\title{
Emittance growth due to misalignment in multistage laser-plasma accelerators
}

\author{
Maxence Thévenet," Rémi Lehe, Carl B. Schroeder, Carlo Benedetti, \\ Jean-Luc Vay, Eric Esarey, and Wim P. Leemans \\ Lawrence Berkeley National Laboratory, Berkeley, California 94720, USA
}

(Received 6 December 2018; published 28 May 2019)

\begin{abstract}
Beam degradation is examined in a laser-plasma accelerator stage with a parabolic plasma channel when the laser pulse and/or the electron beam enters the channel off axis. Betatron oscillations in the beam become incoherent, resulting in a net increase of beam emittance through phase mixing. A quantitative model for transverse emittance growth due to misalignment in multistage accelerators, valid in the linear regime, is presented and compared with particle-in-cell simulations. The model is applied to a chain of laser-plasma accelerator stages, and tolerances are derived on the initial energy spread of the electron beam and misalignment in the multistage structure, with repercussions in high-energy physics applications of laser-plasma accelerators.
\end{abstract}

DOI: 10.1103/PhysRevAccelBeams.22.051302

\section{INTRODUCTION}

Progress made in the past decades allowed laser-plasma accelerators (LPAs) to create electron beams with increasing energy and brightness [1-5]. Such accelerators are seen as potential alternatives to conventional accelerators to build a lepton collider, where the desired energy is on the order of $1 \mathrm{TeV}$ [6]. While a single LPA can accelerate an electron beam by a few $\mathrm{GeV}$, accelerating a high-quality electron beam to $\mathrm{TeV}$ energy with a single LPA requires a driver laser pulse energy that is not achievable with current technology. A solution to reach high energy is to build an accelerator by cascading tens of LPA stages [7-9] (a method called staging), each of them accelerating the electron beam by up to $10 \mathrm{GeV}$.

A collider requires particle beams with not only high energy, but also high quality. Emittance is a quantity that measures the beam quality and, in particular, its focusability [10]. Emittance is a critical parameter for colliders, where the required number of collisions strongly depends on the achieved beam spot size. Emittance is also relevant for most particle beam applications, ranging from medical imaging to $\mathrm{x}$-ray or gamma-ray generation using an LPA-driven free-electron laser or betatron oscillations inherently present in LPAs [11-13]. Hence, a number of

\footnotetext{
"mthevenet@lbl.gov

Present address: Deutsches Elektronen-Synchrotron DESY, 22607 Hamburg, Germany.

Published by the American Physical Society under the terms of the Creative Commons Attribution 4.0 International license. Further distribution of this work must maintain attribution to the author(s) and the published article's title, journal citation, and DOI.
}

theoretical and experimental studies have been dedicated to understand and limit beam degradation during beam trapping or acceleration.

Emittance growth has been a vast field of study in the community of conventional accelerators, and sources of emittance growth range from nonlinearities in the applied forces to instabilities and collisions [14]. Misalignment of one or several beam optics elements results in emittance growth through emittance dilution, and Ref. [15] contains an expression for the emittance after full dilution. However, the emittance does not always reach full dilution within an LPA stage. For this reason, we derive the emittance saturated value and its growth rate in the presence of acceleration, which has not been published yet to the best of our knowledge.

Several studies describe emittance growth in LPAs. Emittance growth due to beam transport between stages has been studied in Refs. [10,16]. Emittance growth due to betatron frequency mismatch when the electron beam has a finite duration has been studied in Ref. [17]. The present article focuses on emittance growth due to laser or beam transverse misalignment at the entrance of an LPA stage. Such a misalignment can result from laser pointing fluctuations, which is a well-known issue in the Ti:sapphire laser technology $[18,19]$. Furthermore, recent work has shown that laser imperfections, e.g., pulse front tilt, can also lead to electron beam or laser pulse deflection at the end of an LPA stage, thus resulting in transverse misalignment in the consecutive stage [20].

In this article, we present a model of transverse emittance growth due to transverse misalignment of the electron beam and/or the laser pulse driver in a plasma channel with a parabolic transverse density profile, where electron acceleration is taken into account. In the presence of a finite 
energy spread in the electron beam, phase mixing of betatron oscillations results in emittance growth. This effect strongly depends on the initial misalignment of the electron beam. Furthermore, laser misalignment results in transverse oscillations of the laser pulse centroid [21], which may lead to emittance growth in the accelerated beam. The model assumes that the LPA is operated in the quasilinear regime with a large plasma channel and that betatron oscillations are not resonant with transverse laser centroid oscillations. Beam loading, cavitation, or ion motion effects are not considered.

Section II describes the typical LPA stage used in this study as well as considerations on beam emittance. Section III provides the theory for emittance growth due to laser pulse or electron beam misalignment, assuming beam-loading effects can be neglected. This model is, in principle, applicable to any type of accelerator provided the assumptions in Section II are satisfied. Section IV shows particle-in-cell (PIC) simulation results and a comparison with the theory. A mitigating solution is proposed in Sec. V to reduce emittance growth due to laser pulse misalignment. Finally, the impact on the design of LPAs is discussed in Sec. VI. Connections between this work and the study of emittance growth in conventional accelerators are briefly discussed in the conclusion.

\section{LPA STAGE AND REQUIREMENTS}

This section describes the typical LPA stage used throughout this article. A $15 \mathrm{~J}$ Gaussian laser pulse with wavelength $\lambda_{0}=0.81 \mu \mathrm{m}$ and duration $\tau_{L}=68 \mathrm{fs}$ [where the temporal profile of the electric field is $\alpha \exp \left(-t^{2} / \tau_{L}^{2}\right)$, equivalent to $80 \mathrm{fs}$ full width at half maximum (FWHM) in intensity] propagates in the $z$ direction and is focused down to a vacuum waist $w_{0}=53 \mu \mathrm{m}$. The Système International system is used throughout this article. The normalized vector potential $a_{0}$, defined as $a_{0}=e A / m_{e} c$, where $e$ is the elementary charge, $A$ is the laser vector potential, $m_{e}$ is the electron mass, and $c$ is the speed of light, reaches the value of 1.38 at focus.

The plasma is a $30-\mathrm{cm}$-long capillary with a preformed parabolic transverse density profile to avoid laser pulse diffraction $[4,22]$. The density profile is radially symmetric, and the electron density is given by

$$
n_{e}(r)=n_{0}\left(1+\frac{4}{k_{p 0}^{2}} \frac{r^{2}}{R_{c}^{4}}\right),
$$

where $r$ is the radial coordinate, $n_{0}$ is the plasma density on axis that is equal to $2.2 \times 10^{17} \mathrm{~cm}^{-3}, R_{c}$ is the capillary matched radius, which is set to $R_{c}=70 \mu \mathrm{m}$ hereafter, and $k_{p 0}=\left[4 \pi n_{0} e^{2} /\left(m_{e} c^{2}\right)\right]^{1 / 2}$ is the plasma wave number on axis. The plasma wave number at any point in space is given by $k_{p}=k_{p 0}\left(n_{e} / n_{0}\right)^{1 / 2}$. This profile is shown in Fig. 1(a). The plasma wavelength $\lambda_{p}$ and plasma frequency $\omega_{p}$ are $\omega_{p}=2 \pi c / \lambda_{p}=k_{p} c$, and their value on axis is
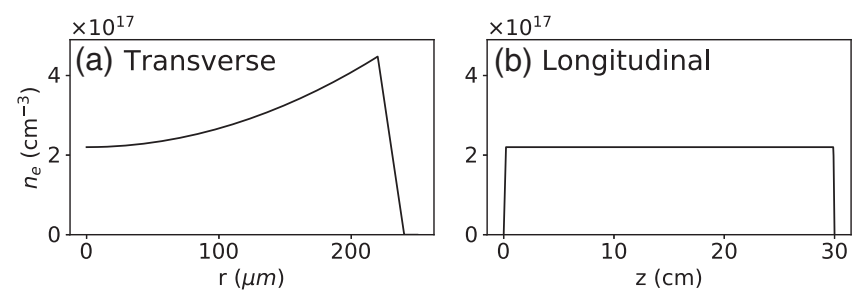

FIG. 1. (a) Transverse and (b) longitudinal profiles for the 30 -cm-long capillary. The longitudinal profile is given on axis.

denoted with subscript $0: \lambda_{p 0}$ and $\omega_{p 0}$. The longitudinal profile is flat with a $2 \mathrm{~mm}$ linear up ramp at the entrance of the capillary and a $1 \mathrm{~mm}$ linear down ramp at the capillary exit, which are common values for these experiments and were observed to have a minimal effect in the energy range studied. The laser pulse is focused at the beginning of the density plateau, and the ramps were kept smaller than the Rayleigh length of the laser $z_{R} \simeq 11 \mathrm{~mm}$. This profile is shown in Fig. 1(b).

An electron beam is externally injected in the LPA stage with initial energy $\mathcal{E}=4.5 \mathrm{GeV}$ and is accelerated up to $8.5 \mathrm{GeV}$, so this setup represents a $4 \mathrm{GeV}$ LPA stage. The electron beam is injected in the second plasma period behind the laser driver to avoid interaction with the laser pulse. The beam has a uniform distribution in the longitudinal direction, with length $L_{\text {beam }}=8 \mu \mathrm{m}$ and a transverse Gaussian distribution with width $\sigma_{x, y}=0.8 \mu \mathrm{m}$ ( $\sigma$ stands for rms size throughout the article). The beam has a Gaussian distribution in longitudinal and transverse momenta with divergence $\sigma_{\theta}=0.11 \mathrm{mrad}$, and the energy spread is $\Delta \mathcal{E} / \mathcal{E}=2.5 \%$. The beam total charge is small enough for beam loading to be negligible $(<1 \mathrm{fC})$ in this study. For an electron beam with transverse Gaussian distribution and peak density $n_{b}$, this condition reads $k_{p}^{3} \sigma_{x} \sigma_{y} L_{\text {beam }} n_{b} / n_{0} \ll 1$. Though beam loading is expected to affect the wakefield amplitude experienced by the electron beam in an LPA-based collider, we expect the main conclusions of this study to be valid in the presence of weak beam loading. All laser pulse, plasma, and beam parameters are summarized in Table I. Apart from the beam charge, these are typical parameters used for LPA experiments on the Petawatt system [23] of the BELLA (BErkeley Laboratory Laser Accelerator) center at the Lawrence Berkeley National Laboratory.

Beam quality is described in terms of the rms (rootmean-square) normalized emittance as defined in Ref. [10] by

$$
\epsilon_{x}=\sqrt{\left\langle x^{2}\right\rangle\left\langle u_{x}^{2}\right\rangle-\left\langle x u_{x}\right\rangle^{2}},
$$

where $x$ is a transverse direction, $u_{x}$ is the normalized momentum in the $x$ direction defined from the momentum as $u_{x}=p_{x} /\left(m_{e} c\right)$, and \langle\rangle stands for average over particles in the beam. Here, the beam is assumed to propagate along 
(a)

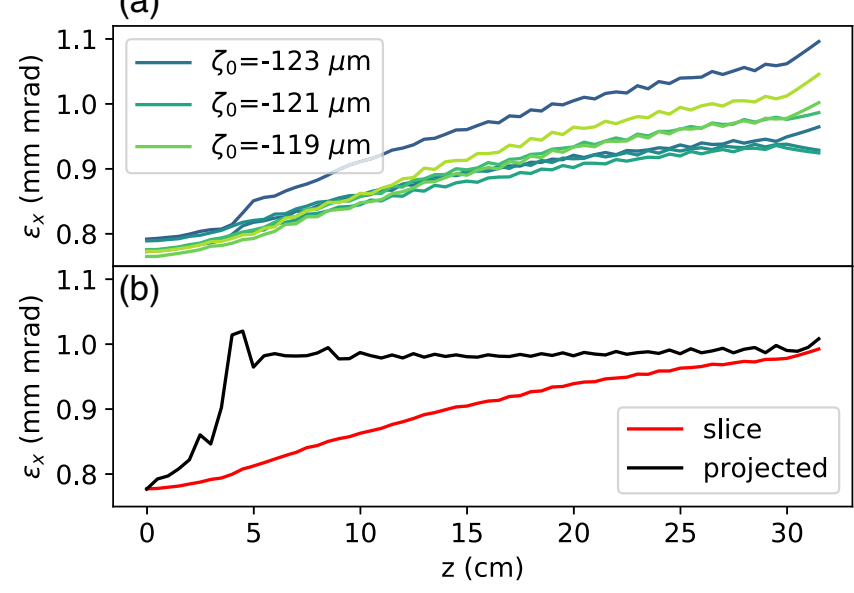

FIG. 2. Beam emittance along propagation when the laser pulse and the electron beam propagate along the capillary axis. (a) Emittance for several beam slices (from blue $\zeta_{0}=$ $-123 \mu \mathrm{m}$ to green $\zeta_{0}=-119 \mu \mathrm{m}$ ). (b) Projected emittance (black) and slice emittance (red). The simulation parameters are given in Table I. The emittance grows from 0.8 to $1.0 \mathrm{~mm}$ mrad, showing that the beam is not perfectly matched.

the $z$ axis. The normalized emittance is relevant within accelerator stages, as it remains constant if all forces are linear and if each beam slice is matched; i.e., the beam has no correlation in $x$ and $u_{x}$, and $\sigma_{x}=\left\langle x^{2}\right\rangle^{1 / 2}$ and $\sigma_{u_{x}}=$ $\left\langle u_{x}^{2}\right\rangle^{1 / 2}$ verify $\epsilon_{x}=\sigma_{x} \sigma_{u_{x}}$ (where the averaging is done within a slice of the beam), even when the accelerated beam has a finite energy spread. Figure 2(a) shows the emittance for several longitudinal $1-\mu \mathrm{m}$-thick slices of the electron beam along propagation in the LPA in a particle-in-cell simulation.

Particle-in-cell simulations were performed with the code Warp [24,25], in a boosted frame [26] with Lorentz factor $\gamma_{\text {boost }}=20$, giving an expected speedup of 400. The longitudinal resolution was $\Delta z=\lambda_{0} / 25$, and the transverse resolution was $\Delta x, y=\lambda_{0} / 0.6$. The physical box dimensions were $500 \mu \mathrm{m} \times 500 \mu \mathrm{m} \times 220 \mu \mathrm{m}$, with one particle per cell for both electrons and ions in the plasma and $2 \times 10^{5}$ particles in the accelerated electron beam. Simulations used the Boris pusher, finite-difference time domain Cole-Karkkainen solver [27] with Cowan coefficients [28], and third-order shape factor for current deposition and field gather. The time step was set to the Courant condition for the Cole-Karkkainen field solver $\Delta t=\Delta z / c$. Numerical Cherenkov instability was mitigated by applying a specific filter [29].

Figure 2(b) shows the projected emittance and the emittance of each slice averaged over the full beam (hereafter called the slice emittance) along propagation from a Warp simulation. Applying Eq. (2) on the whole beam provides the projected emittance. It gives the overall beam quality, but it may strongly depend on the beam length, as the head and the tail of the beam experience different
TABLE I. Simulation parameters. The first column contains laser pulse parameters: wavelength $\lambda_{0}$, energy $\mathcal{E}_{\text {laser, }}$ duration $\tau_{L}$, initial waist $w_{0}$, and normalized amplitude $a_{0}$. The laser is polarized in the $y$ direction. The second column contains electron beam parameters: energy $\mathcal{E}_{\text {beam }}$, length $L_{\text {beam }}$, widths $\sigma_{x, y}$, energy spread $\Delta \mathcal{E} / \mathcal{E}$, and divergence $\sigma_{\theta}$. The third column contains the plasma parameters: density $n_{0}$, stage length $L_{\text {plasma }}$, channel radius $R_{c}$, plasma wavelength $\lambda_{p 0}$, and plasma width in the capillary $w_{c}$. Widths are given as rms values, assuming Gaussian distributions for all quantities.

\begin{tabular}{lccccc}
\hline \hline Laser & \multicolumn{4}{c}{ Beam } & \multicolumn{2}{c}{ Plasma } \\
\hline$\lambda_{0}$ & $0.81 \mu \mathrm{m}$ & $\mathcal{E}_{\text {beam }}$ & $4.5 \mathrm{GeV}$ & $n_{0}$ & $2.2 \times 10^{17} \mathrm{~cm}^{-3}$ \\
$\mathcal{E}_{\text {laser }}$ & $15 \mathrm{~J}$ & $L_{\text {beam }}$ & $8 \mu \mathrm{m}$ & $L_{\text {plasma }}$ & $30 \mathrm{~cm}$ \\
$\tau_{L}$ & $68 \mathrm{fs}$ & $\sigma_{x, y}$ & $0.8 \mu \mathrm{m}$ & $R_{c}$ & $70 \mu \mathrm{m}$ \\
$w_{0}$ & $53 \mu \mathrm{m}$ & $\Delta \mathcal{E} / \mathcal{E}$ & $2.5 \%$ & $\lambda_{p 0}$ & $71 \mu \mathrm{m}$ \\
$a_{0}$ & 1.4 & $\sigma_{\theta}$ & $0.11 \mathrm{mrad}$ & $w_{c}$ & $200 \mu \mathrm{m}$ \\
\hline \hline
\end{tabular}

accelerating and focusing forces in the quasilinear regime. The slice emittance is obtained by applying Eq. (2) on each slice of the beam and averaging the result. The thickness of each slice is taken small enough $(1 \mu \mathrm{m})$ so that the final result does not depend on the thickness of each slice. The slice emittance provides the best achievable beam quality. In practice, it means that reshaping the beam after the accelerating stage can reduce its projected emittance down to its slice emittance at best. Hence, the rest of this article focuses on the slice emittance. By construction, the projected emittance is always larger than the slice emittance, as illustrated in Fig. 2(b). The model presented in Sec. III considers electrons located in the same slice, so that variations of the wave number of betatron oscillations $k_{\beta}$ inside the bunch due to finite length are not considered.

Section III presents a model for emittance growth due to misalignment in an accelerator stage without beam loading. The model is confirmed with 3D PIC simulations in Sec. IV, using the physical parameters presented in Table I.

\section{THEORY FOR EMITTANCE GROWTH WITHOUT BEAM LOADING}

Figure 3 shows snapshots of the $x-u_{x}$ phase space of the beam central slice for different propagation distances for an electron beam with initial transverse offset $X_{0}=5 \mu \mathrm{m}$, where $x=0$ stands for the capillary axis. The laser propagates along the $z$ axis. It shows that phase mixing within a slice is responsible for emittance growth in these conditions: Electrons with different initial energies perform betatron oscillations with different frequencies, leading to decoherence and emittance growth (the same simulation without energy spread shows a negligible energy spread). The betatron period for this slice is $\lambda_{\beta} \simeq$ $3 \mathrm{~cm}$ at the entrance of the capillary. Note that electrons within the same slice undergo the same accelerating and 


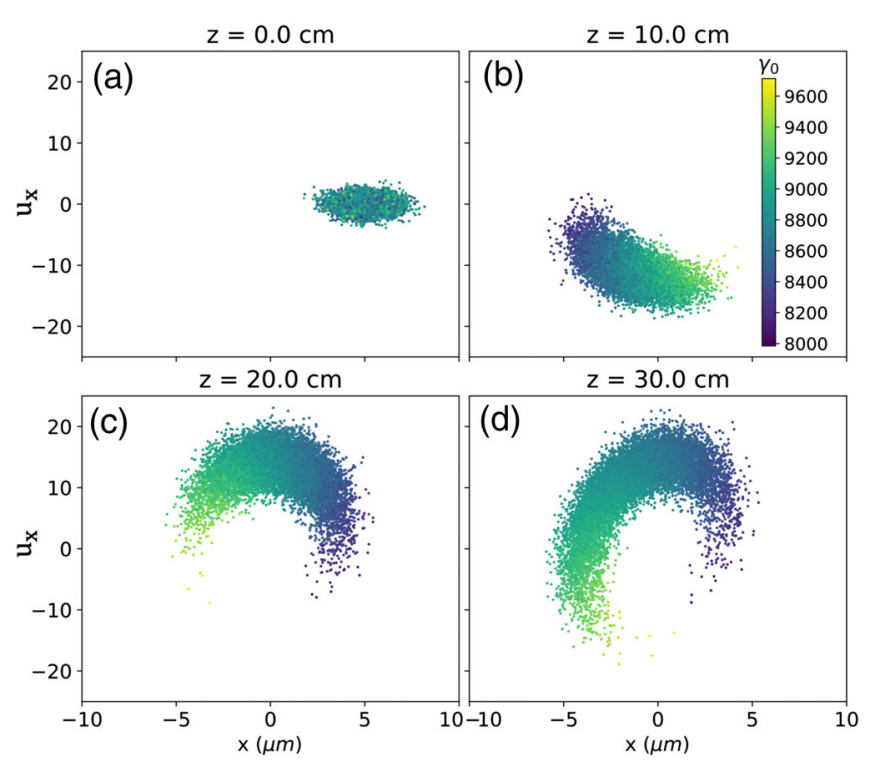

FIG. 3. Phase diagram $x-u_{x}$ at different $z$ along propagation. Simulation conditions are given in Table I. The laser pulse propagated on axis, and the electron beam started with an initial $5 \mu \mathrm{m}$ transverse offset. Colors represent the electron initial Lorentz factor.

focusing fields, so the absolute energy spread $\Delta \mathcal{E} \simeq$ $0.11 \mathrm{GeV}$ remains constant along propagation.

When the laser pulse is injected off axis, another effect can lead to emittance growth. Let $\bar{x}$ be the transverse position of the laser pulse. Figure 4 shows the evolution of $\bar{x}$ along the propagation for various initial laser offsets $\bar{x}_{0}$, ranging from 0 to $30 \mu \mathrm{m}$. The pulse centroid performs harmonic oscillations with period $\bar{\lambda}=2 \pi^{2} R_{c}^{2} / \lambda_{0}$ (here $\bar{\lambda} \simeq 12 \mathrm{~cm}$ ) independent of the initial offset, consistent with Ref. [21]. The frequency of centroid oscillations is $\bar{\omega}=2 \pi c / \bar{\lambda}$.

Several mechanisms responsible for emittance growth are present when both the laser pulse and the electron beam are off axis: (i) The energy spread in the electron beam causes betatron oscillations to decohere, which leads to emittance growth. (ii) Accelerated electrons follow the laser, so the electron beam performs the same slow transverse oscillations as the laser pulse, with period $\bar{\lambda}$. This can lead to emittance growth when these oscillations are not

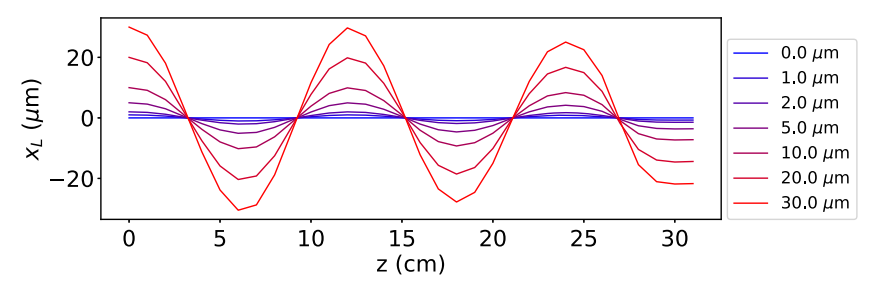

FIG. 4. Laser transverse position $\bar{x}_{0}$ along propagation from Warp simulations. Colors represent different initial transverse positions between 0 and $30 \mu \mathrm{m}$. Simulation conditions are given in Table I. adiabatic, i.e., when condition $\bar{\lambda} \gg \lambda_{\beta}$ is not satisfied (see below). (iii) The plasma density in the capillary is nonhomogeneous transversally, which breaks the symmetry of the laser wake. We hereafter derive an expression for emittance evolution along propagation by considering these three factors. In this range of parameters, radiation damping is small enough to be negligible [30].

Let $\bar{x}_{0}$ be the initial laser offset in the transverse direction $x$. All considerations are given in the $x-z$ plane for simplicity. The transverse force in the linear regime is due to the electric field $E_{x}$, which can be calculated as in Ref. [31]. Assuming that the laser offset remains small, $\bar{x}(t) \ll k_{p 0} R_{c}^{2}$, where $k_{p 0}$ is the electron plasma frequency on the capillary axis, and the electron displacement remains small, $|x-\bar{x}| \ll w_{0}$, the transverse electric field behind the laser pulse (i.e., in a region where the laser field is zero) and close to the laser axis is $E_{x}=E_{x}^{(0)}+E_{x}^{(1)}$ with

$$
\begin{aligned}
& E_{x}^{(0)}(x, \zeta)=\alpha \frac{m_{e} c^{2}}{e} a_{0}^{2} \frac{(x-\bar{x})}{w^{2}} \sin \left(k_{p 0} \zeta\right), \\
& E_{x}^{(1)}(x, \zeta)=-\alpha \frac{m_{e} c^{2}}{e} a_{0}^{2} \frac{\bar{x} \zeta}{k_{p 0} R_{c}^{4}} \cos \left(k_{p 0} \zeta\right),
\end{aligned}
$$

where only the two first-order terms $E_{x}^{(0)}$ (transverse field in a uniform plasma) and $E_{x}^{(1)}$ (first-order correction, i.e., keeping the linear gradient term in a Taylor expansion around $\bar{x}$ ) were kept. The derivation of Eqs. (3) and (4) is presented in Appendix A. Here, $\alpha=\sqrt{\pi / 2} \omega_{p 0} \tau_{L} e^{-\omega_{p 0}^{2} \tau_{L}^{2} / 8}$ is the coupling coefficient between the Gaussian laser length and the plasma wavelength, $\zeta=z-c t$ is the copropagating variable (with $\zeta=0$ the longitudinal position of the laser pulse centroid), and $w$ is the pulse width. We also define

$$
\kappa=1+\frac{\zeta w_{0}^{2}}{k_{p 0} R_{c}^{4} \tan \left(k_{p 0} \zeta\right)},
$$

which represents the field deformation of the wake due to the transverse density gradient when the laser propagates off-axis. The second term on the right-hand side (rhs) originates from $E_{x}^{(1)} / E_{x}^{(0)}$. A more accurate description of the fields when the laser propagates along the axis of a capillary can be found in Ref. [32]. In particular, the derivation in Ref. [32] includes the plasma wave front curvature due to the radial dependence of the plasma wavelength, which was neglected in this study as we consider small displacements around the capillary axis.

\section{A. Emittance growth without acceleration}

In this section, the beam is assumed to travel with constant energy within a stage, i.e., $\left(\Gamma_{f}-\Gamma_{0}\right) / \Gamma_{0} \ll 1$, where $\Gamma_{0}$ and $\Gamma_{f}$ are the initial and final average Lorentz 
factors for the electron beam, respectively. For a $1 \mathrm{TeV}$ accelerator with 100 identical $10 \mathrm{GeV}$ stages, this approximation is valid after $\simeq 10$ stages. The emittance growth rate and saturated value are derived for a slice of an electron beam. While derived in the context of an LPA stage, this model is, in principle, applicable to other types of accelerators $[33,34]$ with constant accelerating field $E_{z}$ and a linear transverse focusing force associated with betatron frequency $\omega_{\beta}$, centered around $\bar{x}_{0} \cos (\bar{\omega} t)$.

The equation for the transverse position $x$ of an electron with Lorentz factor $\gamma=\gamma_{0}$ and betatron frequency $\omega_{\beta}=$ $\omega_{\beta 0}$ in the laser wake is that of a driven harmonic oscillator:

$$
x^{\prime \prime}+\omega_{\beta}^{2} x=\omega_{\beta}^{2} \kappa \overline{x_{0}} e^{i \bar{\omega} t},
$$

where prime symbols stand for time derivative and $\omega_{\beta}$ is the betatron frequency given by

$$
\omega_{\beta}^{2}=\frac{\alpha a_{0}^{2} c^{2}}{\gamma w^{2}} \sin \left(k_{p 0} \zeta\right)
$$

The solution of Eq. (6) is

$$
\begin{aligned}
x= & \left(x_{0}-\eta \kappa \bar{x}_{0}\right) \cos \left(\omega_{\beta} t\right)+\frac{u_{x 0} c}{\gamma \omega_{\beta}} \sin \left(\omega_{\beta} t\right) \\
& +\eta \kappa \bar{x}_{0} \cos (\bar{\omega} t),
\end{aligned}
$$

where the first two rhs terms stand for betatron oscillations and the third rhs term describes the transverse oscillations of the laser pulse centroid. The additional parameter $\eta$ was introduced:

$$
\eta=\frac{\omega_{\beta}^{2}}{\omega_{\beta}^{2}-\bar{\omega}^{2}}
$$

and represents the coupling between betatron oscillations and transverse oscillations of the laser pulse centroid.

Let us consider a slice of an electron beam, into which all electrons experience the same accelerating and focusing fields. Average quantities are denoted with capital letters, and rms quantities are denoted by $\sigma$; e.g., $X$ is the average transverse position of the electron beam and $\sigma_{x}$ is its width. Furthermore, subscript 0 stands for initial values at the entrance of the stage, and \langle\rangle stands for the average over all electrons in the slice: $\Gamma=\langle\gamma\rangle, \Omega_{\beta}=\left\langle\omega_{\beta}\right\rangle$.

From the equation of motion for one electron in the plasma fields, one can follow the procedure used in the Appendix of Ref. [30] and readily derive $u_{x}, X, U_{x}$, $\left\langle\delta x^{2}\right\rangle^{1 / 2},\left\langle\delta u_{x}^{2}\right\rangle^{1 / 2}$, and $\left\langle\delta x \delta u_{x}\right\rangle^{1 / 2}$, where $\delta x=x-X$ and $\delta u_{x}=u_{x}-U_{x}$. The emittance is given by $\epsilon_{x}=$ $\sqrt{\left\langle\delta x^{2}\right\rangle\left\langle\delta u_{x}^{2}\right\rangle-\left\langle\delta x \delta u_{x}\right\rangle^{2}}$.

The mechanism for emittance growth is phase mixing, which comes up when betatron oscillations of electrons decohere because of the difference in betatron frequency. While each electron performs oscillations with constant amplitude, the average beam transverse position tends towards the laser axis because of phase mixing. With the Gaussian distribution in $\gamma$ considered here, one can compute the averaging $\left\langle\cos \left(\omega_{\beta} t\right)\right\rangle$ assuming the relative energy spread is small. The phase term in transverse oscillations of the electron beam

$$
\left\langle\cos \left(\omega_{\beta} t\right)\right\rangle=e^{-\nu_{0}^{2} t^{2}} \cos \left(\Omega_{\beta} t\right)
$$

shows phase mixing leading to $\left\langle\cos \left(\omega_{\beta} t\right)\right\rangle \rightarrow 0$, and the phase mixing rate $\nu_{0}$ is given by

$$
\nu_{0}=\frac{\Omega_{\beta} \sigma_{\gamma}}{2 \sqrt{2} \Gamma} .
$$

Assuming the electron beam is focused at the entrance of the stage, its emittance grows exponentially from its initial value $\epsilon_{x 0}=\sigma_{x 0} \sigma_{u_{x} 0}$ to its saturated value given by

$$
\epsilon_{x, \mathrm{sat}}=\frac{\Omega_{\beta} \Gamma}{2 c}\left[\left(X_{0}-H \kappa \bar{x}_{0}\right)^{2}+\sigma_{x_{0}}^{2}+\left(\frac{c \sigma_{u_{x 0}}}{\Gamma \Omega_{\beta}}\right)^{2}\right],
$$

where $H=\langle\eta\rangle$ and the term $H \kappa \bar{x}_{0}$ represents laser displacement. The emittance growth rate is $\propto 2 \nu_{0}$ [the factor of 2 comes from the term in Eq. (10), squared to compute the emittance].

When the beam has no correlations and is matched at the entrance, i.e., $\epsilon_{0}=\sigma_{x} \sigma_{u_{x} 0}$, the emittance does not grow even in the presence of energy spread, provided all fields are linear and vary slowly and there is no misalignment. Further considerations are given in Sec. VI. The matching condition for the beam width and divergence reads

$$
\sigma_{x}=\left(\frac{\epsilon_{x} c}{\Gamma \Omega_{\beta}}\right)^{1 / 2}, \quad \sigma_{u_{x}}=\left(\frac{\Gamma \epsilon_{x} \Omega_{\beta}}{c}\right)^{1 / 2} .
$$

When the matching condition is met, the saturated emittance is simply expressed in terms of the initial emittance as

$$
\frac{\epsilon_{x, \text { sat }}}{\epsilon_{x 0}}=1+\frac{1}{2}\left(\frac{X_{0}-H \kappa \bar{x}_{0}}{\sigma_{x 0}}\right)^{2} .
$$

Equations (11) and (12) give the main features of emittance growth, namely, the saturated value and the growth rate. On the one hand, Eq. (12) shows that the acceptable misalignment is on the order of the beam width if one wants to avoid dramatic beam degradation. On the other hand, Eq. (11) shows that the growth rate is proportional to the relative energy spread $\sigma_{\gamma} / \Gamma$ : The emittance grows faster in the presence of a large energy spread. From Eq. (11), one can readily show that emittance growth remains small in a plasma with length $L$ provided $\sigma_{\gamma} / \Gamma \ll \lambda_{\beta} / L$.

These equations give the behavior of emittance growth in most stages of a multistage LPA and illustrate the involved mechanisms as well as the role of beam properties. However, it relies on the strong assumption that the 
betatron frequency and energy of the electrons remain constant in the whole stage. A more general model for emittance growth, that is also valid in the first stages, is derived in the following paragraphs.

\section{B. Emittance growth with acceleration}

The equation of motion for an electron in the accelerator stage with fields given by Eqs. (3) and (4) reads

$$
x^{\prime \prime}+\frac{\gamma^{\prime}}{\gamma} x^{\prime}+\omega_{\beta}^{2} x=\omega_{\beta}^{2} \kappa \overline{x_{0}} e^{i \bar{\omega} t} .
$$

The orbit of an electron in these fields (see Appendix B) is

$$
\begin{aligned}
x(t)= & +\left(\frac{\gamma_{0}}{\gamma}\right)^{1 / 4}\left[x_{0}-\eta \kappa \bar{x}_{0}\right] \cos \left(\int \omega_{\beta}\right) \\
& +\left(\frac{\gamma_{0}}{\gamma}\right)^{1 / 4} \frac{u_{x 0} c}{\gamma_{0} \omega_{\beta}} \sin \left(\int \omega_{\beta}\right)+\eta \kappa \bar{x}_{0} \cos (\bar{\omega} t), \\
u_{x}(t)= & -\left(\frac{\gamma_{0}}{\gamma}\right)^{1 / 4}\left[x_{0}-\eta \kappa \bar{x}_{0}\right] \gamma \frac{\omega_{\beta}}{c} \sin \left(\int \omega_{\beta}\right) \\
& +\left(\frac{\gamma_{0}}{\gamma}\right)^{1 / 4} u_{x 0} \cos \left(\int \omega_{\beta}\right)-\gamma \frac{\bar{\omega}}{c} \eta \kappa \bar{x}_{0} \sin (\bar{\omega} t),
\end{aligned}
$$

where $\int \omega_{\beta}$ is a shorthand for $\int_{0}^{t} \omega_{\beta}(s) d s$, where $s$ stands for time. These expressions are exact in the WKB approximation when $\bar{x}_{0}=0$. When the laser is off axis $\left(\bar{x}_{0} \neq 0\right)$, laser centroid oscillations are assumed to be much slower than betatron oscillations, so that $\eta \simeq 1$. Also assumed is

$$
\bar{\omega} \frac{\gamma^{\prime}}{\gamma} \ll\left|\omega_{\beta}^{2}-\bar{\omega}^{2}\right| .
$$

This nonresonance assumption is similar to the WKB approximation for a driven harmonic oscillator (see Appendix B). We emphasize that this is a strong assumption when studying acceleration up to $1 \mathrm{TeV}$, as $\omega_{\beta} \gg \bar{\omega}$ in the first stages while $\omega_{\beta} \ll \bar{\omega}$ in the last stages. However, as shown in the last section of this article, emittance growth occurs in the first few stages of a chain of accelerators, where this approximation is valid.

An electron beam is assumed to have Gaussian distributions in position transversally ( $x$ and $y$ ) and momentum $\left(u_{x}, u_{y}\right.$, and $\left.u_{z}\right)$, and the same notations are used as in the previous section. The beam still has a uniform distribution longitudinally. The beam is assumed to have a narrow energy spread $\sigma_{\gamma} \ll \Gamma$. The beam width $\sigma_{x}=\left\langle x^{2}\right\rangle^{1 / 2}$, normalized transverse momentum spread $\left\langle u_{x}^{2}\right\rangle^{1 / 2}$, and position-momentum correlation $\left\langle x u_{x}\right\rangle$ along the $x$ direction required to calculate the beam emittance $\epsilon_{x}$ are, respectively, given by

$$
\begin{aligned}
& 2\left(\frac{\gamma}{\gamma_{0}}\right)^{1 / 2}\left\langle\delta x^{2}\right\rangle=+\left[\left(X_{0}-H \kappa \bar{x}_{0}\right)^{2}+\sigma_{x 0}^{2}\right] \times\left[1+\cos \left(2 \int \Omega_{\beta}\right) e^{-4 \nu^{2} t^{2}}\right] \\
& +\frac{c^{2} \sigma_{u_{x 0}}^{2}}{\Gamma_{0}^{2} \Omega_{\beta 0}^{2}} \times\left[1-\cos \left(2 \int \Omega_{\beta}\right) e^{-4 \nu^{2} t^{2}}\right]-\left(X_{0}-H \kappa \bar{x}_{0}\right)^{2} e^{-2 \nu^{2} t^{2}} \times\left[1+\cos \left(2 \int \Omega_{\beta}\right)\right], \\
& 2\left(\frac{\gamma}{\gamma_{0}}\right)^{1 / 2} \frac{c^{2}}{\gamma^{2} \Omega_{\beta}^{2}}\left\langle\delta u_{x}^{2}\right\rangle=+\left[\left(X_{0}-H \kappa \bar{x}_{0}\right)^{2}+\sigma_{x 0}^{2}\right] \times\left[1-\cos \left(2 \int \Omega_{\beta}\right) e^{-4 \nu^{2} t^{2}}\right] \\
& +\frac{c^{2} \sigma_{u_{x 0}}^{2}}{\Gamma_{0}^{2} \Omega_{\beta 0}^{2}} \times\left[1+\cos \left(2 \int \Omega_{\beta}\right) e^{-4 \nu^{2} t^{2}}\right]-\left(X_{0}-H \kappa \bar{x}_{0}\right)^{2} e^{-2 \nu^{2} t^{2}} \times\left[1-\cos \left(2 \int \Omega_{\beta}\right)\right], \\
& 2\left(\frac{\gamma}{\gamma_{0}}\right)^{1 / 2} \frac{c}{\gamma \Omega_{\beta}}\left\langle\delta x \delta u_{x}\right\rangle=-\left[\left(X_{0}-H \kappa \bar{x}_{0}\right)^{2}+\sigma_{x 0}^{2}\right] \times \sin \left(2 \int \Omega_{\beta}\right) e^{-4 \nu^{2} t^{2}} \\
& +\frac{c^{2} \sigma_{u_{x 0}}^{2}}{\Gamma_{0}^{2} \Omega_{\beta 0}^{2}} \times \sin \left(2 \int \Omega_{\beta}\right) e^{-4 \nu^{2} t^{2}}+\left(X_{0}-H \kappa \bar{x}_{0}\right)^{2} e^{-2 \nu^{2} t^{2}} \times \sin \left(2 \int \Omega_{\beta}\right),
\end{aligned}
$$

where

$$
\nu(t)=\frac{1-(1+\tau)^{-1 / 2}}{\sqrt{2} \tau} \frac{\Omega_{\beta 0} \sigma_{\gamma_{0}}}{\Gamma_{0}}
$$

with

$$
\tau=\frac{\Gamma_{0}^{\prime} t}{\Gamma_{0}}
$$

The saturated value for the emittance is

$$
\epsilon_{x, \mathrm{sat}}=\frac{\Omega_{\beta 0} \Gamma_{0}}{2 c} \times\left[\left(X_{0}-H \kappa \bar{x}_{0}\right)^{2}+\sigma_{x_{0}}^{2}+\left(\frac{c \sigma_{u_{x 0}}}{\Gamma_{0} \Omega_{\beta 0}}\right)^{2}\right],
$$

which is the same expression as in the previous case Eq. (12) with initial values of the average Lorentz factor and betatron frequency. One can verify that Eq. (11) can be derived from Eq. (20) in the limit $\tau \rightarrow 0$. 
All the above expressions for $x, u_{x},\left\langle\delta x^{2}\right\rangle,\left\langle\delta u_{x}^{2}\right\rangle$, and $\left\langle\delta x \delta u_{x}\right\rangle$ were compared to results from a test-particle code assuming a constant accelerating force and constant linear focusing force and show perfect agreement when the laser is on axis. Good agreement is also found when the nonresonance assumption Eq. (18) is not strictly satisfied. This procedure could also be applied to derive the projected emittance.

Overall, in the presence of misalignment, the emittance grows from initial value $\epsilon_{x 0}$ to saturated value $\epsilon_{x, \text { sat }}$ with growth rate $\nu(t)$. The saturated emittance depends on the distance between the laser axis and beam axis. The growth rate is proportional to the relative energy spread $\sigma_{\gamma} / \Gamma$, and the emittance reaches a significant fraction of the saturated value at the end of a plasma stage with length $L$ if $\sigma_{\gamma} / \Gamma \gtrsim \lambda_{\beta} / L$.

\section{NUMERICAL SIMULATIONS}

This section presents results of PIC simulations of the physical conditions of Sec. II. It provides a comparison with expressions presented in Sec. III. In particular, PIC simulations are presented where (i) the laser pulse is on axis and the electron beam is off axis $\left(\bar{x}_{0}=0, X_{0} \neq 0\right)$ and (ii) the laser pulse is off axis and the electron beam is aligned with the laser pulse $\left(\bar{x}_{0} \neq 0, X_{0}=\bar{x}_{0}\right)$. Note that emittance degradation due to the plasma inhomogeneity was enhanced in this study by injecting the electron beam in the second bucket of the wake and can be reduced by injecting the beam into the first bucket. This enhancement can be understood by considering the factor $\zeta$ in Eq. (4), which is $\simeq \lambda_{p 0}$ in the first bucket and $\simeq 2 \lambda_{p 0}$ in the second bucket.

\section{A. Electron beam off axis}

Figures 5(a) and 5(b) show, respectively, the projected and slice emittance along propagation in the same conditions as Fig. 2, i.e., in a typical $\mathrm{GeV}$-scale LPA stage, from PIC simulations. The final emittance for a beam initially on axis is $\epsilon_{x} \simeq 1 \mathrm{~mm} \mathrm{mrad,} \mathrm{and} \mathrm{transverse} \mathrm{mis-}$ alignment can have dramatic impact: A $1 \mu \mathrm{m}(5 \mu \mathrm{m})$ offset results in a $2 \times(10 \times)$ factor on the final emittance degradation. Similar trends are observed for projected and slice emittance. Theoretical predictions are shown in Fig. 5(c), in fairly good agreement with the PIC simulation results for a transverse offset up to $10 \mu \mathrm{m}$, where simulations show that the transverse fields depend linearly on $x$. For larger offsets $x_{0}=20-30 \mu \mathrm{m}$, the emittance grows faster in the PIC simulations, because the beam interacts with regions where the transverse fields are nonlinear, which is not described in the model.

For simplicity, in computing the bunch emittance starting from the second-order moments given by Eqs. (19), several approximations were made. First, $E_{x}^{(0)}$ and $E_{x}^{(1)}$ were evaluated at their peak value, i.e., neglecting dependency

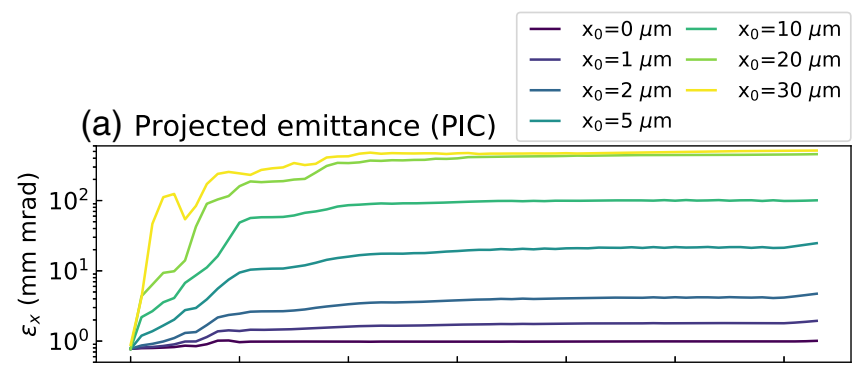

(b) Slice emittance (PIC)

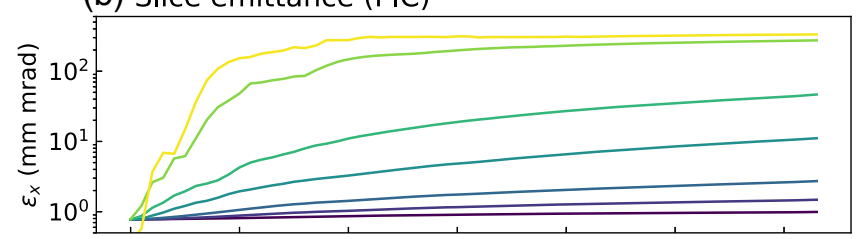

(c) Slice emittance (theory)

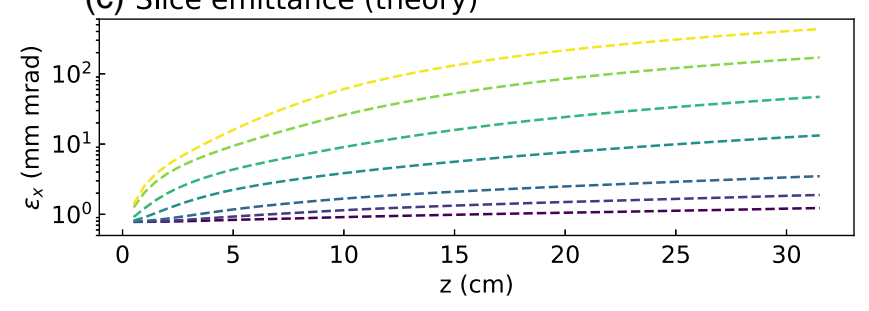

FIG. 5. Emittance growth along propagation due to electron beam misalignment. The initial transverse beam offset $X_{0}$ between 0 and $30 \mu \mathrm{m}$. The laser pulse propagates on axis. Simulation conditions are given in Table I. (a)-(c) show the projected emittance in PIC simulations, slice emittance in PIC simulations, and theoretical predictions for the slice emittance, respectively.

on $\zeta$. The model does not include two effects that occur in the simulation: (i) The electron beam experiences longitudinal dephasing with respect to the wake, and (ii) the laser pulse width oscillates around the matched value, which in turn results in oscillations of the wake structure as well as laser width $w$ and amplitude $a_{0}$. Both effects are neglected in the theory, and the electron beam is assumed to experience constant forces, taken from the tightest focus in the simulations $a_{0}=1.4$ and $w=45 \mu \mathrm{m}$. Furthermore, the interaction takes place in the quasilinear regime, and magnetic force is neglected (the magnetic force is 5 times smaller than the electric force in this study). The absolute energy spread within each slice is assumed to be constant to its initial value, and the beam mean energy $\Gamma$ is taken from the simulation.

Even with these approximations, the model reproduces well the evolution of slice emittance along propagation as long as $X_{0}<10 \mu \mathrm{m}$. For higher values of initial displacement $X_{0}$, the theory breaks down, as $|x-\bar{x}| \ll w_{0}$ is no longer valid. Note that here we examined a higher offset than what is achievable in experiments, where the electron beam and the laser pulse can routinely satisfy $\left|X_{0}\right|,\left|\bar{x}_{0}\right| \lesssim(1-5) \mu \mathrm{m}$, where the theory gives very good predictions. 


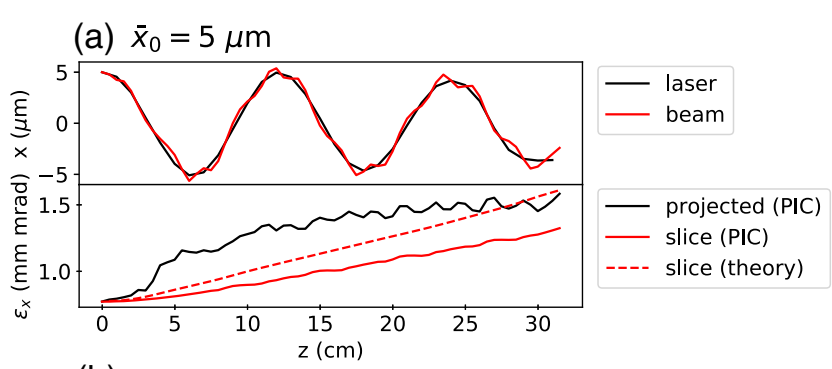

(b) $\bar{x}_{0}=20 \mu \mathrm{m}$

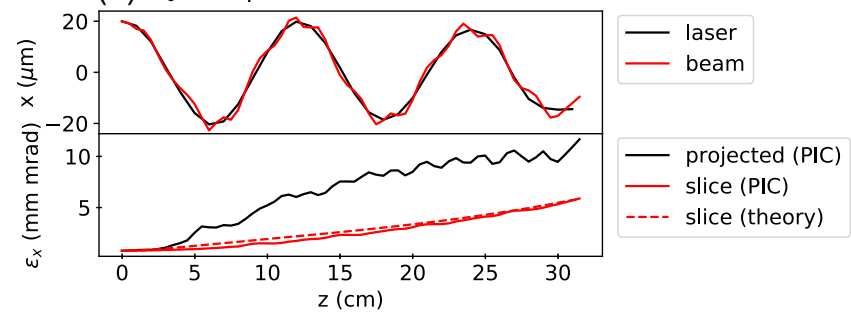

FIG. 6. Emittance growth along propagation due to laser pulse misalignment. (a) The top panel shows the transverse position of the laser pulse (black line) and electron beam (red line). The initial pulse offset is $\bar{x}_{0}=5 \mu \mathrm{m}$, and the electron beam starts with $X_{0}=\bar{x}_{0}$. The electron beam follows the laser centroid oscillations. The bottom panel shows the projected and slice emittance along with the theory for slice emittance. (b) shows the same quantities for $\bar{x}_{0}=X_{0}=20 \mu \mathrm{m}$.

\section{B. Laser pulse off axis}

The case where the laser is off axis and the electron beam starts with the same offset, namely, $\bar{x}_{0}=5 \mu \mathrm{m}$ and $X_{0}=\bar{x}_{0}$, is considered in Fig. 6(a). This is of interest, because it gives the lowest final emittance achievable for a given laser offset. PIC simulations performed showed that $X_{0} \neq \bar{x}_{0}$ results in a higher final emittance than $X_{0}=\bar{x}_{0}$ for a given $\bar{x}_{0}$. Hence, this gives a lower bound to emittance growth for a given laser offset. The top subplot in Fig. 6(a) shows the transverse position of the laser pulse centroid and the average transverse position of the electron beam and shows that the beam follows the laser pulse oscillations (here, $\omega_{\beta} / \bar{\omega} \simeq 5$ ). The bottom subplot in Fig. 6(a) shows the emittance growth in these conditions. Figure 6(b) shows the same plots for a larger initial offset $\bar{x}_{0}=20 \mu \mathrm{m}$. Here, again, the theory reproduces well the slice emittance. In both cases, the emittance growth is mostly due to the field deformation [factor $\kappa$ in Eq. (12)]. Note that Eq. (12) suggests that emittance growth when the laser pulse is off axis could be considerably reduced by satisfying the condition $X_{0}=\eta \kappa \bar{x}_{0}$. This is, however, not achievable in a realistic case, as $\kappa$ evolves significantly in the presence of longitudinal dephasing with respect to the wakefield.

\section{Resonance, when $\omega_{\beta} \simeq \overline{\boldsymbol{\omega}}$}

Equation (9) shows that $\eta$ diverges when the resonance condition is satisfied $\omega_{\beta}=\bar{\omega}$, i.e., when the betatron frequency equals the frequency of laser centroid transverse

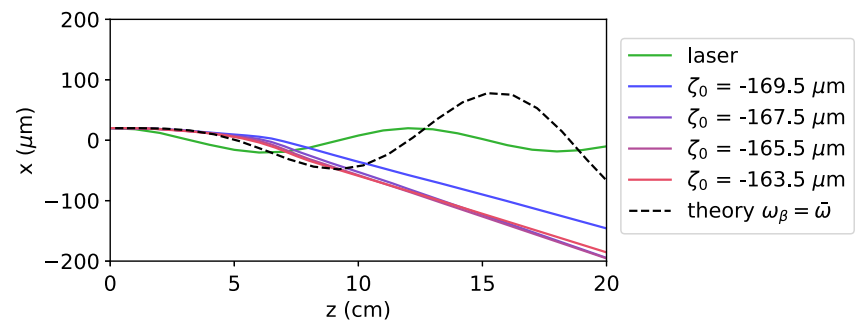

FIG. 7. Beam resonance with $\omega_{\beta} \simeq \bar{\omega}$. The green line shows the laser pulse centroid position, and other lines show the transverse position of different slices of the electron beam. The black dashed line shows the theory for the beam position when the resonance condition is met. Simulation conditions are given in Table I, except the initial beam energy $\mathcal{E}_{\text {beam }}=156 \mathrm{GeV}$. The initial transverse position of the laser pulse and electron beam is $\bar{x}_{0}=X_{0}=20 \mu \mathrm{m}$.

oscillations. In this case, one can solve the equations of motion for an electron [Eq. (15)] assuming the beam energy is constant, $x(t) \propto t \sin \left(\omega_{\beta} t\right)$, such that the amplitude of transverse oscillations grows linearly. An example is shown in Fig. 7, where the laser and plasma parameters were kept constant and the electron Lorentz factor was increased to roughly satisfy the resonance condition. One can see that the electron beam starts to resonate and escapes the wakefield after half an oscillation (the model assumes the wake has an infinite width and does not capture the beam escape, which explains the discrepancy for $z>10 \mathrm{~cm})$. This is somehow an extreme case, as the initial offset of $20 \mu \mathrm{m}$ is much greater than what is expected in near-future high-repetition rate laser facilities.

\section{Effect of energy spread}

Figure 8 shows the emittance growth for several values of the initial energy spread. As expected, the higher the initial energy spread, the faster the convergence to the saturated emittance value, when each slice has reached full decoherence. Hence, full decoherence is reached after $15 \mathrm{~cm}$ for a $10 \%$ energy spread and after $30 \mathrm{~cm}$ for a 5\% energy spread. Again, the model reproduces well the trends observed in the simulations.

\section{E. Emittance growth in $\boldsymbol{x}$ and $\boldsymbol{y}$ directions}

Previous considerations were given for electron beam and laser pulse misalignment in the $x$ direction only. In the model derivation, $\epsilon_{x}$ is assumed to depend on misalignment in the $x$ direction only, and, accordingly, $\epsilon_{y}$ is assumed to depend on misalignment in the $y$ direction only. The lack of coupling between orthogonal transverse directions is shown in Fig. 9, in the presence of both laser pulse and electron beam misalignment. Laser misalignment is introduced in the $x$ direction: $\bar{x}_{0}=10 \mu \mathrm{m}$. In Fig. 9(a), two electron beams are injected with $X_{0}=10$ and $12 \mu \mathrm{m}$, respectively, and $Y_{0}=0 \mu \mathrm{m}$. Beams have different $X_{0}$; hence, they have different final emittance in the $x$ direction $\epsilon_{x}$. 
(a) Projected emittance

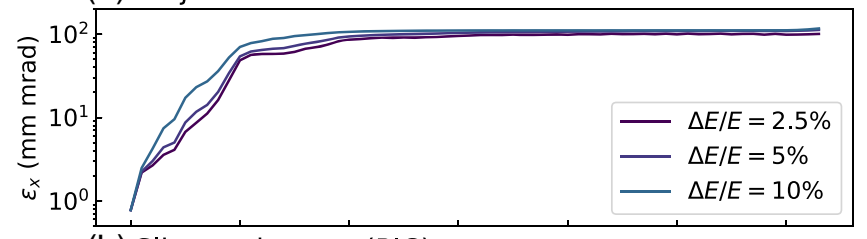

(b) Slice emittance (PIC)

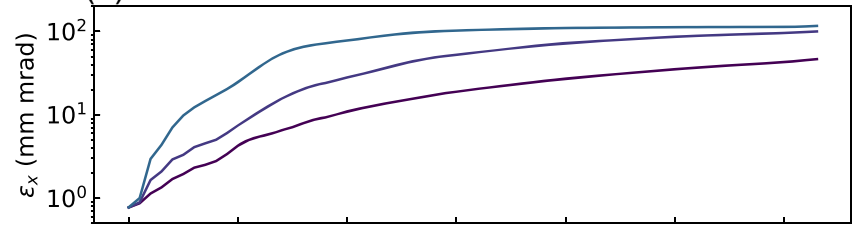

(c) Slice emittance (theory)

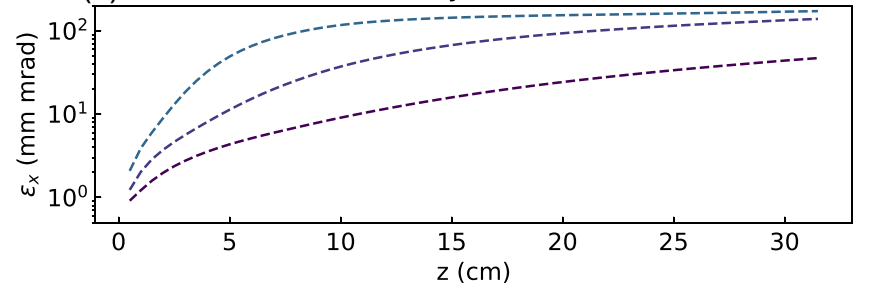

FIG. 8. Emittance growth for various energy spreads. Simulation conditions are given in Table I. The laser pulse propagates on axis, and the electron beam starts with a $10 \mu \mathrm{m}$ transverse offset. (a)-(c) show, respectively, the PIC projected emittance, the PIC slice emittance, and the theory for slice emittance. The energy spread is $2.5 \%, 5 \%$, and $10 \%$, respectively.

Yet, they have the same $Y_{0}$, and they have the same $\epsilon_{y}$. Note that $\epsilon_{y}$ is the same for $\bar{x}_{0}=0 \mu \mathrm{m}$ and $\bar{x}_{0}=10 \mu \mathrm{m}$. Figure 9(b) shows similar results, where electron beams have the same $X_{0}$ and different $Y_{0}$. In this case, both beams have

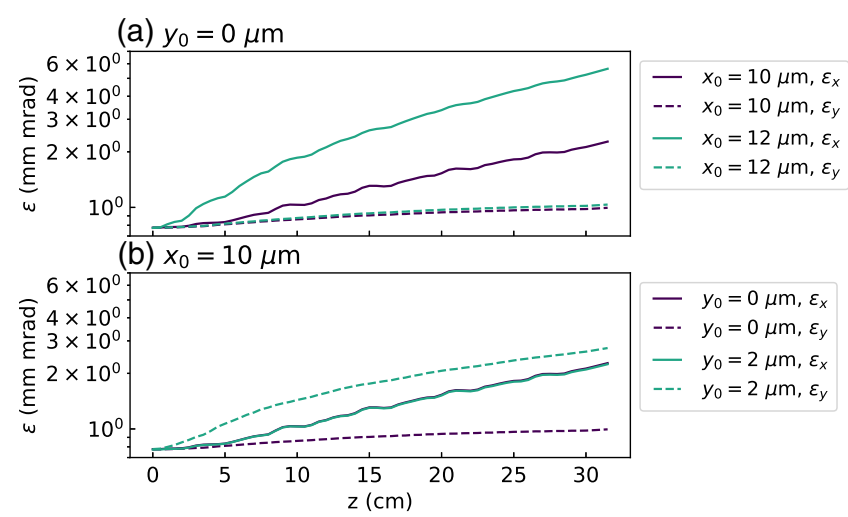

FIG. 9. Growth of slice emittance $\epsilon_{x}$ and $\epsilon_{y}$ in both transverse directions $x$ and $y$ along propagation in the presence of laser pulse and electron beam misalignments from Warp simulations. The simulation conditions are given in Table I. The laser pulse initial position is $\bar{x}_{0}=10 \mu \mathrm{m}$. (a) Two electron beams start with $X_{0}=$ $10 \mu \mathrm{m}$ and $X_{0}=12 \mu \mathrm{m}$, respectively, with $Y_{0}=0 \mu \mathrm{m}$. The $\epsilon_{y}$ emittance is the same for both beams. (b) shows the same result for beams with the same $X_{0}=10 \mu \mathrm{m}$ and with $Y_{0}=0$ and $2 \mu \mathrm{m}$, respectively. In this case, both beams have the same $\epsilon_{x}$ emittance evolution. the same $\epsilon_{x}$ and different $\epsilon_{y}$. As could be expected from noncoupling of transverse coordinates, emittance growth in the $x$ and $y$ directions is independent. Hence, the model presented in Sec. III can be used along direction $x$ or $y$ independently.

\section{PASSIVE PLASMA LENS TO MITIGATE EMITTANCE GROWTH DUE TO MISALIGNMENT}

Using Eq. (12), the saturated emittance, for $\nu t \gg 1$, is

$$
\epsilon_{x, \mathrm{sat}}=\frac{\Omega_{\beta} \Gamma}{2 c}\left(X_{0}-\eta \kappa \bar{x}_{0}\right)^{2},
$$

where the contributions of the beam width and divergence were neglected, i.e., $\sigma_{x, 0} \ll X_{0}, \bar{x}_{0}$ and $c \sigma_{\theta, 0} / \Omega_{\beta} \ll X_{0}, \bar{x}_{0}$. Moreover, nonlinearities in the transverse field are assumed to be small, $E_{x}^{(1)} \ll E_{x}^{(0)}$, so that $\eta \simeq 1$ and $\kappa \simeq 1$. In this case, the saturated value for the emittance is proportional to the distance between the initial laser pulse and the initial electron beam positions $\left|X_{0}-\bar{x}_{0}\right|$. Hence, minimal emittance growth is obtained when the electron beam enters the stage behind the laser $X_{0}=\bar{x}_{0}$, as was studied in Fig. 6. For a given laser offset, the emittance growth can be reduced by adiabatically (i.e., with displacements much slower than the betatron frequency) positioning the electron beam behind the laser pulse.

We propose to use a passive plasma lens [35,36] at the entrance of the plasma stage to position the electron beam on the laser pulse axis. A low-density few-millimeter plasma jet was introduced before the accelerator stage to serve as a plasma lens: The transverse force in the laser wake kicks the electron beam to bring it close to the laser axis. For the sake of simplicity, this scheme is tested with a collimated electron beam $\sigma_{\theta, 0}=0$. The plasma lens density was scanned, and the effect of the plasma lens is shown in Fig. 10. The optimal density does not depend on the initial transverse displacement. The left column shows the beam transverse position, the projected emittance, and the slice emittance along propagation without a plasma lens, and the right column shows the same quantities when a plasma lens was used. Different colors represent for different initial beam positions while the laser pulse starts on axis. The topright panel shows that the electron beam is brought on axis by the plasma lens. As the transverse force in the wake is linear, the position at which the electron beam reaches $x=0$ does not depend on the initial position. Using a plasma lens significantly reduces the emittance growth for all initial transverse positions. More specifically, in the absence of misalignment $\left(x_{0}=0\right)$, the plasma lens focuses the electron beam at the entrance of the plasma channel, which reduces the emittance [second term in square brackets in the rhs of Eq. (12)]. In the presence of misalignment, the dominant term in Eq. (12) is the first term in the rhs square bracket; using a plasma lens reduces this term and improves the emittance. An alternative is to 


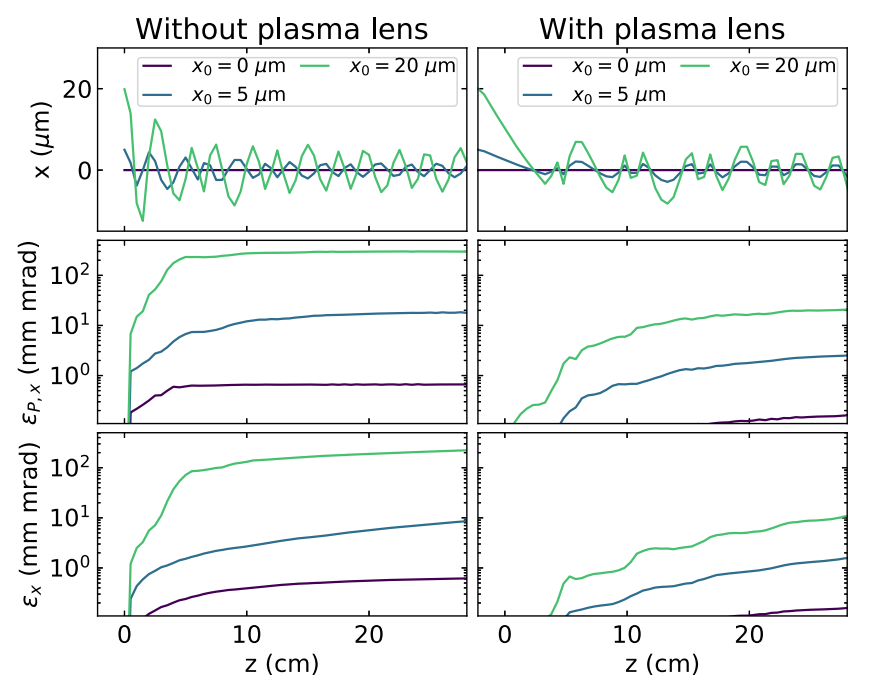

FIG. 10. Beam properties along propagation without a plasma lens (left) and with a plasma lens (right) from Warp simulations. Subplots show, from top to bottom, the transverse position, projected emittance, and sliced emittance. This image shows numerical results only; the model is not applied here. Colors stand for various initial beam transverse positions. Simulation conditions are given in Table I, where the plasma starts at $z=2 \mathrm{~cm}$. The plasma lens has an eighth-order super-Gaussian longitudinal profile with center $z_{\text {lens }}=2 \mathrm{~mm}$, length $L_{\text {lens }}=$ $2.9 \mathrm{~mm}$ FWHM, and peak density $n_{\text {lens }}=5.5 \times 10^{15} \mathrm{~cm}^{-3}$.

taper the entrance of the LPA stage with a smooth plasma density ramp.

\section{IMPACT FOR A 1 TEV STAGED LPA}

In this section, we consider the impact of misalignment on the design of a staged LPA made of $N$ identical stages of acceleration with $\Delta \mathcal{E}=m_{e} c^{2} \Delta \Gamma$ each. The emittance at the entrance of each stage is assumed to be the one at the end of the previous stage, and the beam is matched at the entrance of each stage.

The exact evolution of the beam properties depends on a large number of parameters. The beam emittance would, in particular, depend on the beam evolution between stages, e.g., the propagation distance in free space as well as potential focusing optics, which are neglected in the scope of this work, as well as ion motion [37,38], beam or laser hosing, and cavitation. Since emittance growth in the $x$ and $y$ directions is independent, as shown in Fig. 9, beam displacement is considered in the $x$ direction.

\section{A. A TeV staged LPA with 1-m stages}

In this section, the impact of beam misalignment on the design of a $1 \mathrm{TeV}$ electron accelerator made of 100 stages of $10 \mathrm{GeV}$ accelerators is investigated. The laser and plasma parameters are those used in Table I, except the plasma length $L_{\text {stage }}=1 \mathrm{~m}$, the initial beam energy is $10 \mathrm{GeV}$, and the laser normalized amplitude is $a_{0}=1$. The initial beam emittance is $10^{-3} \mathrm{~mm}$ mrad. The characteristic length for emittance growth due to misalignment is given by $L_{\text {growth }}=c / 2 \nu$, and the saturated value of emittance is given by $\epsilon_{x, \text { sat }}=\Omega_{\beta} \Gamma X_{0}^{2} /(2 c)$.

Results are shown in Fig. 11. Figure 11(b) shows $L_{\text {growth }}$ within each stage. As the beam energy increases, the characteristic length for emittance growth becomes increasingly large, growing much larger than the stage length, after
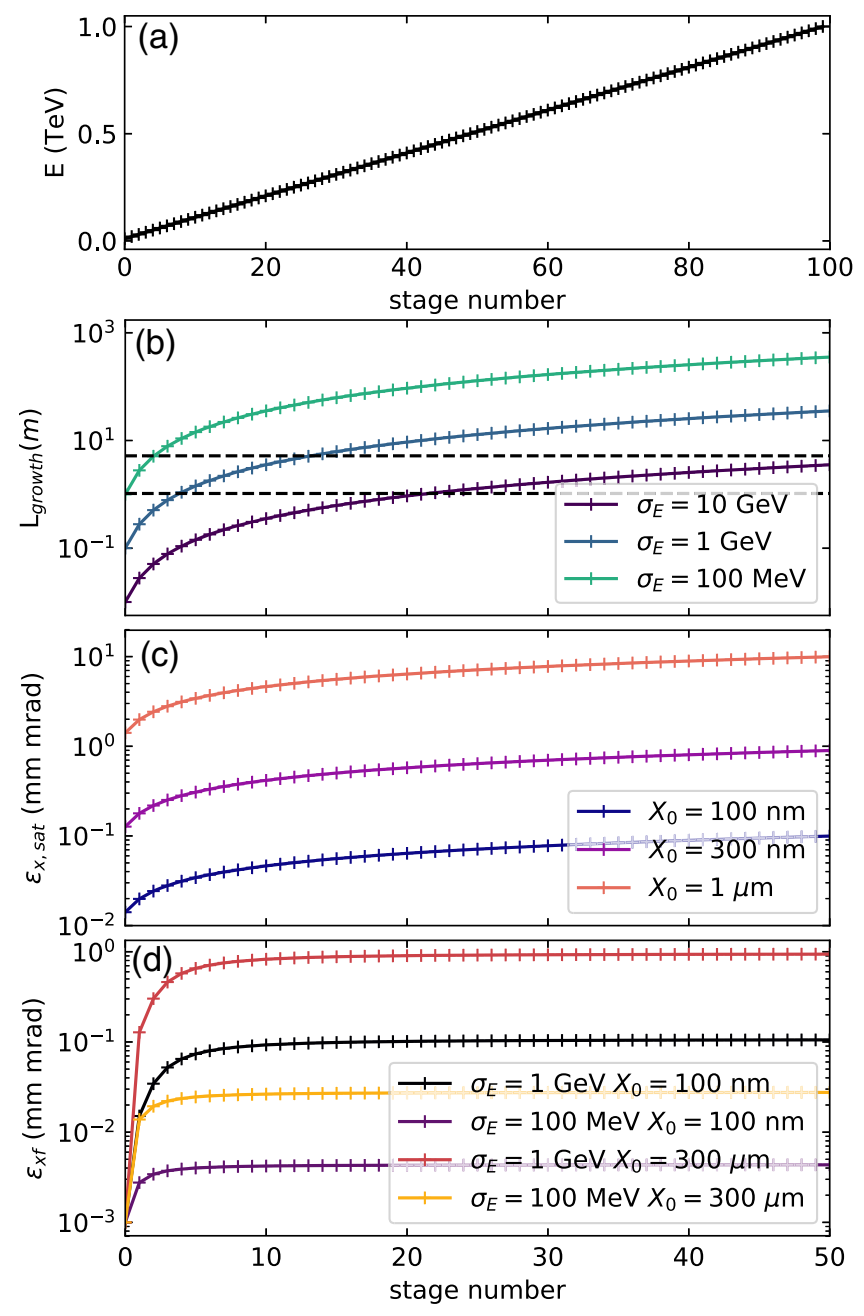

FIG. 11. Effect of misalignment on each stage of a $1 \mathrm{TeV}$ collider for a beam with initial emittance $\epsilon_{x 0}=10^{-3} \mathrm{~mm} \mathrm{mrad}$. (a) Energy at the end of each stage. (b) Characteristic length for emittance growth due to phase mixing in the presence of misalignment within each stage $L_{\text {growth }}=c / 2 \nu$. Cases of 0.1 , 1 , and $10 \mathrm{GeV}$ energy spread, i.e., $0.01 \%, 0.1 \%$, and $1 \%$ of the final energy, respectively, were considered. The bottom dashed line shows the stage length $L_{\text {stage }}$. The top dashed line shows $5 \times L_{\text {stage }}$. When $L_{\text {growth }}>5 \times L_{\text {stage }}$, the beam is rigid and the emittance does not evolve within each stage. (c) Saturated value for the emittance given by Eq. (23) for different values of initial offsets. The beam enters each stage with the same offset $X_{0}$. The laser propagates on axis. (d) Emittance at the end of each stage given by Eq. (23), for $\bar{x}_{0}=0$ and two values of energy spread. 
which the emittance is roughly constant within each stage. A low energy spread is crucial in the very first stages of a chain of LPAs, as this is where emittance growth occurs.

Figure 11(c) shows the saturated emittance in each stage given by Eq. (23), i.e., the value the beam emittance would reach in each stage if $L_{\text {stage }} \gg L_{\text {growth. }}$. The transverse laser offset was varied between $1 \mu \mathrm{m}$ (achievable for current laser systems) down to $100 \mathrm{~nm}$ (expected for near-future systems with a higher repetition rate [23]).

Again, this plot shows how dramatic beam degradation can be when $L_{\text {growth }}$ is small, i.e., when the relative energy spread is large. Figure 11(d) shows the calculated beam emittance at the entrance of each stage. While beam quality can be preserved with a sufficiently low energy spread, the beam can endure severe degradation with a $1 \mathrm{GeV}$ energy spread, assumed constant along the LPA chain.

Finally, we emphasize that the model for emittance growth when the laser is off axis is valid provided that Eq. (18) is satisfied, which is when the betatron frequency is far from resonance with the frequency of laser transverse centroid oscillations. Assuming $\omega_{p 0} \gg \bar{\omega}$ and considering the first stages of a chained LPA, this condition reads $\Gamma \ll \pi^{2} a_{0}^{2} \omega_{0}^{2} / \omega_{p 0}^{2}$. On the other hand, emittance growth within a stage is negligible provided that $L_{\text {growth }} \gg L_{\text {stage }}$, which can be expressed as $\Gamma \gg \sqrt{4 / 2 \pi a_{0}} \sqrt{\sigma_{\gamma} \Delta \Gamma}$, where $\Delta \Gamma$ is the increase of the beam Lorentz factor within a stage. Combining these equations, and taking $a_{0}=1$, the model can be used to predict emittance growth in the whole chain of LPAs provided that

$$
\sigma_{\gamma} \ll \frac{150}{\Delta \Gamma}\left(\frac{n_{e}}{n_{c}}\right)^{-2} .
$$

With the physical parameters presented in Table I, the condition on the energy spread reads $\sigma_{\mathcal{E}} \ll 300 \mathrm{GeV}$, which is easily satisfied, so that the model can effectively predict emittance growth in the whole chain in the presence of laser misalignment.

\section{B. General considerations}

The matching condition Eq. (13) gives Eq. (14), which can be written as

$$
\epsilon_{x, \text { sat }}=\left(1+\frac{\delta X_{0}^{2}}{2}\right) \epsilon_{x 0},
$$

where $\delta X_{0}$ is the relative misalignment

$$
\delta X_{0}=\frac{X_{0}-\eta \kappa \bar{x}_{0}}{\sigma_{x 0}}
$$

at the entrance of each stage (note that $\sigma_{x 0} \propto \gamma^{-1 / 4}$ depends on the stage, and its final value can be below $100 \mathrm{~nm}$ ). Offsets are assumed to be small, so that field deformation can be neglected $\kappa \simeq 1$ and stages far from resonance are considered, so that $\eta \simeq 1$. In this case, the emittance growth depends only on the relative offset between the laser pulse and the electron beam $X_{0}-\bar{x}_{0}$, which justifies the use of a plasma lens presented in Sec. V.

The emittance evolves from initial value $\epsilon_{x 0}$ to saturation value $\epsilon_{x, \text { sat }}$ as $e^{-\nu^{2} t^{2}}$. Following the scaling laws for the linear regime in Ref. [2] and assuming that (i) the stage length is determined by the dephasing length $L_{\text {stage }}=$ $L_{\text {deph }}=\lambda_{p 0}^{3} /\left(2 \lambda_{0}^{2}\right)$ and (ii) the laser pulse width is of the order of the plasma wavelength $w \simeq \lambda_{p 0}$, the emittance growth within each stage is determined by Eq. (25) and

$$
\frac{L_{\text {stage }}}{L_{\text {growth }}}=\left(\frac{\sqrt{\alpha}}{2 \sqrt{2} \pi a_{0}}\right) \frac{\sigma_{\gamma} \Delta \Gamma}{\Gamma^{3 / 2}} \sim \sigma_{\gamma}^{\text {rel }} \Delta \Gamma^{\text {rel }} \Gamma^{1 / 2},
$$

where $\sigma_{\gamma}^{\text {rel }}=\sigma_{\gamma} / \Gamma$ is the relative energy spread and $\Delta \Gamma^{\text {rel }}=$ $\Delta \Gamma / \Gamma$ is the relative acceleration within each stage. The emittance at the end of the stage $\epsilon_{x f}$ is $\epsilon_{x f}=\epsilon_{x 0}$ if $L_{\text {stage }} \ll$ $L_{\text {growth }}$ and $\epsilon_{x f}=\epsilon_{x \text {,sat }}$ if $L_{\text {stage }} \gg L_{\text {growth }}$. The first factor on the rhs of Eq. (27) is on the order of unity for LPAs in the quasilinear regime.

The beam energy after stage $n$ is $\Gamma_{0}+n \Delta \Gamma$. In the regime $\sigma_{\gamma} \Delta \Gamma \ll \Gamma_{0}^{3 / 2}$, emittance growth occurs mostly in the first stage, and the relative emittance growth within the whole staged LPA is $\lesssim \delta X_{0}^{2}$. This is the regime where a chain of LPAs for a staged LPA would best operate.

In the regime $\sigma_{\gamma} \Delta \Gamma>\Gamma_{0}^{3 / 2}$, emittance growth occurs in several stages, and a simple estimate for the emittance growth in the full accelerator chain is given by $\epsilon_{x f}=n_{\text {sat }} \times \epsilon_{x, \text { sat }}$, where $n_{\text {sat }}$ is the stage number for which $L_{\text {stage }}=L_{\text {growth }}$. Assuming all stages have the same relative offset (alternatively, random error could be assumed), the final emittance is

$$
\epsilon_{x f}=\left(1+\frac{\delta X_{0}^{2}}{2}\right)^{n_{\mathrm{sat}}} \epsilon_{x 0},
$$

with

$$
n_{\mathrm{sat}}=\frac{\sigma_{\gamma}^{2 / 3} \Delta \Gamma^{2 / 3}-\Gamma_{0}}{\Delta \Gamma} .
$$

The model presented in Sec. III was used to predict emittance growth due to misalignment in a given LPA chain operating with $a_{0}=1$ and $L_{\text {stage }}=1 \mathrm{~m}$. Figure 12 shows the final emittance as a function of the absolute transverse offset and the energy spread. The dashed line shows $\epsilon_{x f}=10^{-2} \mathrm{mmmrad}$, which is considered as a reference value at the interaction point [39]. In practice, there is a trade-off between acceptable transverse offset and energy spread: A $1 \mathrm{GeV}$ energy spread requires transverse alignment to a precision of $100 \mathrm{~nm}$, while a $100 \mathrm{MeV}$ energy spread relaxes this constraint to $1 \mu \mathrm{m}$. 


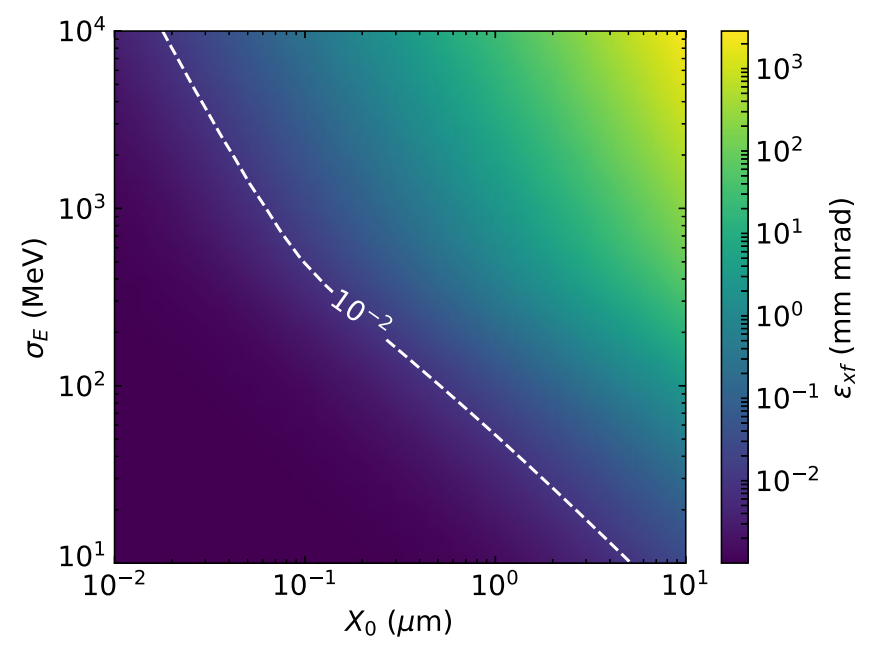

FIG. 12. Model projection for the final transverse emittance at the end of a $1 \mathrm{TeV}$ collider with initial emittance $\epsilon_{x 0}=$ $10^{-3} \mathrm{~mm} \mathrm{mrad}$ as a function of the initial transverse offset and initial absolute energy spread. The initial beam width is $26 \mathrm{~nm}$. Scaling laws presented in Ref. [2] were used, so that using $L_{\text {stage }}=1 \mathrm{~m}$ and $a_{0}=1$ sets all other parameters. The chain contains 71 stages. The initial laser width verifies $w=\lambda_{p 0}$. The initial beam energy is $10 \mathrm{GeV}$. The dashed line shows $\epsilon_{x f}=10^{-2} \mathrm{~mm}$ mrad, which is considered as a reference value at the interaction point [39].

Figure 13 shows the counterpart of Fig. 12: The tolerance on misalignment is shown as a function of the LPA chain parameters (beam initial energy and length of each stage) for given beam parameters at the end of the

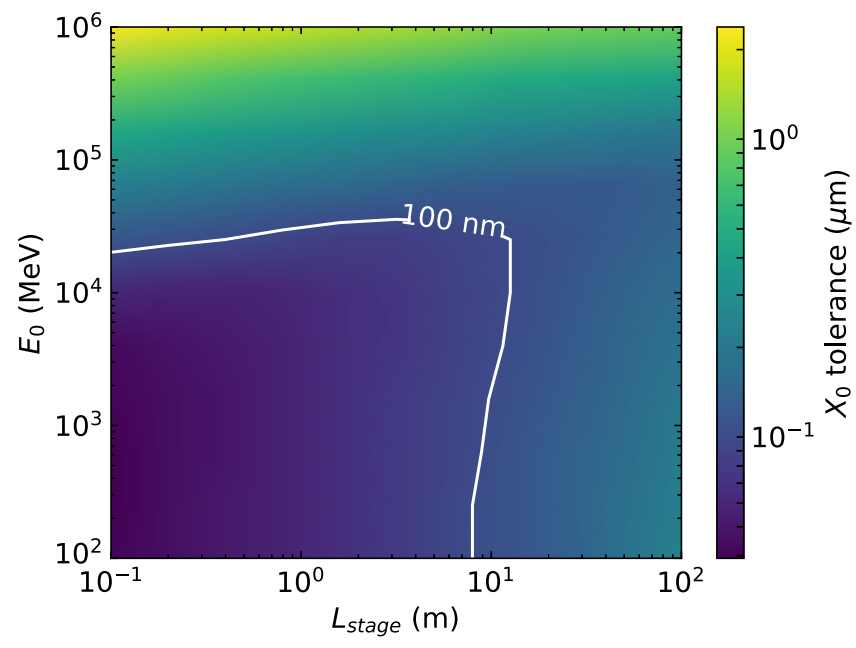

FIG. 13. Model projection for the tolerance on absolute beam displacement assuming the final beam emittance is $\epsilon_{x f}=$ $0.01 \mathrm{~mm} \mathrm{mrad}$ as a function of the stage length and initial energy. The absolute energy spread is constant to $1 \mathrm{GeV}$, and the initial beam emittance is $\epsilon_{x 0}=10^{-3} \mathrm{~mm} \mathrm{mrad}$. Following the scalings in the linear regime for $a_{0} \lesssim 1$ from Ref. [2], the energy gain per stage is $\propto L_{\text {stage }}^{2 / 3}$, so that $L_{\text {stage }}=1 \mathrm{~m}$ corresponds to a chain of 70 LPAs and $L_{\text {stage }}=100 \mathrm{~m}$ corresponds to a chain of four LPAs. chain. Increasing the length of each stage (even up to an extreme value of $100 \mathrm{~m}$ ) has little effect on the tolerance, while increasing the initial energy greatly loosens the requirements on absolute alignment.

\section{DISCUSSION AND CONCLUSIONS}

Several assumptions were made throughout this work and will require further investigations in the design of a staged LPA reaching TeV energy. First, beam loading was neglected, though it will play a role for efficient LPA stages. Second, the beam propagation between two consecutive stages was not considered, while it can lead to emittance growth or be used to insert beam-cleaning optics. Third, the collider model assumed no correlation at the entrance of each stage $\left\langle x u_{x}\right\rangle=0$. Finally, nonlinearities (including electron cavitation and ion motion) were omitted. Within this scope, the model presented above provides a quantitative analysis of beam degradation due to misalignment and emphasizes the importance of beam and laser control in the first few stages. Improving the beam quality may rely on other techniques, including density tapering. Besides, misalignment of laser pulse of electron beam is likely to result in aggravated misalignment in the consecutive stage, so a stabilizing technique may be required. The study presented above focuses on a chain of identical LPAs, but optimization may require varying LPA stage parameters along the chain. Furthermore, developments similar to Sec. III A could be used to assess emittance growth due to misalignment in transport lines between stages, which could also be a significant source of beam degradation in the LPA chain.

This article describes how beam and/or laser misalignment results in emittance growth in the presence of a finite energy spread. This class of effects has been described in the literature of conventional accelerators, in terms of filamentation due to chromaticity. Nevertheless, the main results of this article, the expressions for saturated emittance (22) and growth rate Eq. (20) with beam acceleration, have not been published yet, to the best of our knowledge. Equation (14) of Ref. [15], referring to Ref. [40], describes the saturated emittance in the absence of beam acceleration and is the same as Eq. (14).

As a conclusion, this article presents a detailed analysis of beam degradation occurring in an LPA stage in a plasma channel when the electron beam and/or the laser pulse enters the stage off the plasma channel axis. A model is presented for emittance growth without beam loading and is found to be in good agreement with particle-in-cell simulations. Apart from the electron beam and laser pulse offsets, the energy spread is shown to be a critical parameter, as the emittance growth rate is directly proportional to the energy spread. When the laser pulse enters the LPA stage with an offset, we propose to use a passive laserplasma lens at the entrance of the stage to adiabatically position the electron beam behind the laser pulse, hence 
dramatically reducing the emittance growth in the stage. Finally, the model is applied to estimate the emittance growth due to misalignment in a chain of LPA accelerators, which highlights the requirements on laser pointing stability as well as energy spread.

\section{ACKNOWLEDGMENTS}

The authors acknowledge Anthony Gonsalves for fruitful conversations. We thank Chad Mitchell for sharing his expertise on accelerator physics. This work was supported by the Director, Office of Science, Office of High Energy Physics, of the U.S. Department of Energy under Contract No. DE-AC02-05CH11231 and used the computational facilities at the National Energy Research Scientific Computing Center (NERSC) through the high-impact science at scale program.

\section{APPENDIX A: WAKE DEFORMATION}

In this Appendix, expressions for the fields in the laser wake are derived when the laser pulse propagates off axis in the capillary. All calculations are performed in the $x-z$ plane for simplicity, and the Coulomb gauge is used. The LPA is assumed to operate in the linear regime, so that the transverse force is given by $\boldsymbol{E}_{\perp}=\left(-m_{e} c / e\right) \boldsymbol{\nabla} \psi$, where $\psi$ is the wake pseudopotential $\psi=\tilde{\phi}-a_{z}$, where $\tilde{\phi}$ is the normalized electrostatic potential, obtained from the electrostatic potential $\phi$ by $\tilde{\phi}=e \phi /\left(m_{e} c^{2}\right)$, and $a_{z}$ is the $z$ component of the normalized vector potential. Assuming the channel is wide $\left(w \ll R_{c}\right)$, the pseudopotential is given by

$$
\psi=\alpha \frac{a_{0}^{2}}{4} \sin \left(k_{p} \zeta\right) e^{-2\left(\frac{x-\bar{x}}{w}\right)^{2}},
$$

where $\alpha$ is the coupling coefficient between the laser pulse and the plasma wave given in Sec. III. The plasma channel is assumed to have a parabolic transverse profile given by Eq. (1). Assuming small transverse displacement, i.e., $x, \bar{x} \ll k_{p 0}^{2} R_{c}^{4}$, the transverse electric field $E_{x}$ reads

$$
\begin{aligned}
E_{x}= & -\frac{\alpha m_{e} c^{2} a_{0}^{2}}{4 e}\left[\left(\partial_{x} k_{p}\right) \zeta \cos \left(k_{p} \zeta\right)\right. \\
& \left.-4 \frac{x-\bar{x}}{w^{2}} \sin \left(k_{p} \zeta\right)\right]
\end{aligned}
$$

where $\partial_{x}$ stands for $\partial / \partial x$. A Taylor expansion of $k_{p}$ and $\partial_{x} k_{p}$ near the center of the capillary gives

$$
\begin{aligned}
E_{x}= & -\frac{m_{e} c^{2}}{e} \alpha a_{0}^{2}\left\{\cos \left(k_{p 0} \zeta\right)\left[\frac{x \zeta}{k_{p 0} R_{c}^{4}} \cos \left(\frac{2 x^{2} \zeta}{k_{p 0} R_{c}^{4}}\right)-\frac{x-\bar{x}}{w^{2}} \sin \left(\frac{2 x^{2} \zeta}{k_{p 0} R_{c}^{4}}\right)\right]\right. \\
& \left.-\sin \left(k_{p 0} \zeta\right)\left[\frac{x \zeta}{k_{p 0} R_{c}^{4}} \sin \left(\frac{2 x^{2} \zeta}{k_{p 0} R_{c}^{4}}\right)+\frac{x-\bar{x}}{w^{2}} \cos \left(\frac{2 x^{2} \zeta}{k_{p 0} R_{c}^{4}}\right)\right]\right\} .
\end{aligned}
$$

The approximations $x, \bar{x} \ll w \ll k_{p 0}^{2} R_{c}^{4}$ yield

$$
\begin{aligned}
E_{x}= & -\frac{m_{e} c^{2}}{e} \alpha a_{0}^{2}\left[-\frac{x-\bar{x}}{w^{2}} \sin \left(k_{p 0} \zeta\right)\right. \\
& \left.+\frac{\bar{x} \zeta}{k_{p 0} R_{c}^{4}} \cos \left(k_{p 0} \zeta\right)\right],
\end{aligned}
$$

the result given in Eqs. (3) and (4).

\section{APPENDIX B: ELECTRON ORBITS}

In the linear regime, the equation of motion for an electron in the laser wake is

$$
\frac{d \boldsymbol{u}}{d t}=-\frac{e}{m_{e} c} \boldsymbol{E} .
$$

We hereafter consider the motion of an electron in constant fields so that effects like dephasing are neglected $\zeta=$ const. Prime symbols stand for time derivatives, and the derivation uses complex notations. Assuming (i) the electron travels with $u_{z} \gg 1$ and (ii) the electron travels close to the laser axis $x \ll w$, the equations of motion are

$$
\begin{gathered}
\gamma^{\prime}=-\frac{\alpha a_{0}^{2}}{4} \omega_{p 0} \cos \left(k_{p 0} \zeta\right), \\
x^{\prime \prime}+\frac{\gamma^{\prime}}{\gamma} x^{\prime}+\omega_{\beta}^{2} x=\omega_{\beta}^{2} \kappa \overline{x_{0}} e^{i \bar{\omega} t} .
\end{gathered}
$$

Equation (B2) yields constant acceleration $\gamma^{\prime}=$ const, while Eq. (B3) yields damped (betatron) oscillations (lhs) with a driver at the frequency of transverse laser centroid oscillations (rhs), $\bar{\omega}$.

The homogeneous equation in $\chi$ with $x=\chi e^{i \int \omega_{\beta}}$ is

$$
\begin{aligned}
\chi^{\prime \prime}+ & \left(\frac{\gamma^{\prime}}{\gamma}+2 i \bar{\omega}\right) \chi^{\prime} \\
& +\left(\frac{\gamma^{\prime}}{\gamma} i \bar{\omega}+\omega^{2}-\bar{\omega}^{2}\right) \chi=\omega^{2} \bar{x}_{0} e^{i \bar{\omega} t} .
\end{aligned}
$$

Doing two WKB-like approximations $\gamma^{\prime} / \gamma \ll \bar{\omega}$ and $\bar{\omega} \gamma^{\prime} / \gamma \ll\left|\omega^{2}-\bar{\omega}^{2}\right|$, the first element in each bracket is neglected. Two independent solutions are given by 


$$
\begin{aligned}
& f_{1}(t)=\left(\frac{\gamma_{0}}{\gamma}\right)^{1 / 4} e^{+i \int \omega_{\beta}}, \\
& f_{2}(t)=\left(\frac{\gamma_{0}}{\gamma}\right)^{1 / 4} e^{-i \int \omega_{\beta}} .
\end{aligned}
$$

A solution to Eq. (B3) can be found by inserting a solution of the form $x=x_{1} f_{1}+x_{2} f_{2}$ into the equation. It yields

$$
\begin{gathered}
x_{1}^{\prime} f_{1}^{\prime}+x_{2}^{\prime} f_{2}^{\prime}=\omega_{\beta}^{2} \kappa \overline{x_{0}} e^{i \bar{\omega} t}, \\
x_{1}^{\prime} f_{1}+x_{2}^{\prime} f_{2}=0,
\end{gathered}
$$

with solutions

$$
\begin{gathered}
x_{1}=-\left(\frac{\gamma_{0}}{\gamma}\right)^{1 / 4} \kappa \overline{x_{0}} \frac{\omega_{\beta}}{2\left(\bar{\omega}-\omega_{\beta}\right)} e^{i \bar{\omega} t-i \int \omega_{\beta},} \\
x_{2}=+\left(\frac{\gamma_{0}}{\gamma}\right)^{1 / 4} \kappa \overline{x_{0}} \frac{\omega_{\beta}}{2\left(\bar{\omega}+\omega_{\beta}\right)} e^{i \bar{\omega} t+i \int \omega_{\beta}},
\end{gathered}
$$

and

$$
x=\kappa \overline{x_{0}} \eta e^{i \bar{\omega} t} .
$$

The orbit of an electron is

$$
\begin{aligned}
x= & C_{1}\left(\frac{\gamma_{0}}{\gamma}\right)^{1 / 4} e^{i \int \omega_{\beta}}+ \\
& C_{2}\left(\frac{\gamma_{0}}{\gamma}\right)^{1 / 4} e^{-i \int \omega_{\beta}}+\eta \kappa \overline{x_{0}} e^{i \bar{\omega} t}, \\
u_{x}= & \frac{i \omega_{\beta} C_{1}}{c} \gamma_{0}^{1 / 4} \gamma^{3 / 4} e^{i \int \omega_{\beta}}- \\
& \frac{i \omega_{\beta} C_{2}}{c} \gamma_{0}^{1 / 4} \gamma^{3 / 4} e^{-i \int \omega_{\beta}}+\eta \kappa \frac{i \overline{x_{0}} \bar{\omega} \gamma}{c} e^{i \bar{\omega} t},
\end{aligned}
$$

where $C_{1}$ and $C_{2}$ are constants. Solving for initial conditions $x(t=0)=x_{0}$ and $u_{x}(t=0)=u_{x 0}$ gives Eq. (17). Parts of this demonstration can be found at the beginning of chapter 5 in Ref. [41].

\section{APPENDIX C: BEAM QUANTITIES FOR EMITTANCE}

Starting from the orbits of a single electron Eq. (17), the average properties of a set of electrons given in Eq. (19) are derived in this Appendix. The variables $x, u_{x}, \gamma$, and $\omega_{\beta}$ are the transverse position, transverse normalized momentum, Lorentz factor, and betatron frequency of an electron, respectively. Capital letters $Q=\langle q\rangle$ stand for average over all particles in the beam, where $q$ represents any electron quantity. Notation \langle\rangle stands for the average over the set of electrons. We also use notation $\delta q=q-Q$. The beam is assumed to have Gaussian distributions in position transversally ( $x$ and $y)$ and momentum $\left(u_{x}, u_{y}\right.$ and $\left.u_{z}\right)$, and (rms) width of quantity $q$ is denoted by $\sigma_{q}=\left\langle q^{2}\right\rangle^{1 / 2}$. Initial values are denoted by subscript 0 .

Assuming the accelerating field $E_{z}$ is constant along the propagation of an electron, one has $\gamma(t)=\gamma_{0}+\gamma_{0}^{\prime} t$, where $\gamma_{0}^{\prime}$ is the constant accelerating gradient, and the phase of betatron oscillations can be calculated as

$$
\int_{0}^{t} \omega_{\beta}(T) d T=\int_{0}^{t} \omega_{\beta}(T) d T=\tilde{\omega}_{\beta}(t) t
$$

with

$$
\tilde{\omega}_{\beta}(t)=2 \frac{(1+\tau)^{1 / 2}-1}{\tau} \omega_{\beta 0},
$$

where $\tau=\gamma_{0}^{\prime} t / \gamma_{0}$. All electrons in the set are assumed to experience the same accelerating field $\gamma_{0}^{\prime}=\Gamma_{0}{ }^{\prime}$, so that the absolute energy spread remains constant along propagation $\sigma_{\gamma}=\sigma_{\gamma 0}$.

Furthermore, the energy spread is assumed small $\sigma_{\gamma} \ll \Gamma$. This has two consequences: First, $\tau$ can be approximated as $\tau=\Gamma_{0}^{\prime} t / \Gamma_{0}$, and, second, the $\tilde{\omega}_{\beta}$ distribution remains roughly Gaussian with an average value and rms width given, respectively, by

$$
\begin{gathered}
\tilde{\Omega}_{\beta}=\left\langle\tilde{\omega}_{\beta}\right\rangle=2 \frac{(1+\tau)^{1 / 2}-1}{\tau} \Omega_{\beta 0}, \\
\sigma_{\tilde{\omega}_{\beta}}=\left\langle\tilde{\omega}_{\beta}^{2}\right\rangle^{1 / 2}=\frac{1-(1+\tau)^{-1 / 2}}{\tau} \frac{\Omega_{\beta 0} \sigma_{\gamma_{0}}}{\Gamma_{0}} .
\end{gathered}
$$

One can then calculate phase mixing terms

$$
\begin{aligned}
& \left\langle\cos \int \omega_{\beta}\right\rangle=e^{-\nu^{2} t^{2}} \cos \int \Omega_{\beta}, \\
& \left\langle\sin \int \omega_{\beta}\right\rangle=e^{-\nu^{2} t^{2}} \sin \int \Omega_{\beta},
\end{aligned}
$$

where $\nu=\sigma_{\tilde{\omega}_{\beta}} / \sqrt{2}$ is the phase mixing rate. With these quantities, it is straightforward to derive average values. For example, the average position is given by

$$
\begin{aligned}
X(t)= & \left(\frac{\Gamma_{0}}{\Gamma}\right)^{1 / 4}\left[X_{0}-\eta \kappa \bar{x}_{0}\right] e^{-2 \nu^{2} t^{2}} \cos \int \Omega_{\beta} \\
& +\left(\frac{\Gamma_{0}}{\Gamma}\right)^{1 / 4} \frac{U_{x 0} c}{\Gamma_{0} \Omega_{\beta}} e^{-2 \nu^{2} t^{2}} \sin \int \Omega_{\beta} .
\end{aligned}
$$

Equations (19) are then obtained by computing $\delta x=x-X$, performing the averaging $\left\langle\delta x^{2}\right\rangle$, and repeating for $u_{x}$. 
[1] W. P. Leemans et al., Multi-GeV Electron Beams from Capillary-Discharge-Guided Subpetawatt Laser Pulses in the Self-Trapping Regime, Phys. Rev. Lett. 113 (2014).

[2] E. Esarey, C. Schroeder, and W. Leemans, Physics of laserdriven plasma-based electron accelerators, Rev. Mod. Phys. 81, 1229 (2009).

[3] J. Faure, Y. Glinec, A. Pukhov, S. Kiselev, S. Gordienko, E. Lefebvre, J.-P. Rousseau, F. Burgy, and V. Malka, A laserplasma accelerator producing monoenergetic electron beams, Nature (London) 431, 541 (2004).

[4] C. Geddes, C. Toth, J. Van Tilborg, E. Esarey, C. Schroeder, D. Bruhwiler, C. Nieter, J. Cary, and W. Leemans, High-quality electron beams from a laser wakefield accelerator using plasma-channel guiding, Nature (London) 431, 538 (2004).

[5] S. P. Mangles, C. Murphy, Z. Najmudin, A. G. R. Thomas, J. Collier, A. E. Dangor, E. Divall, P. Foster, J. Gallacher, C. Hooker et al., Monoenergetic beams of relativistic electrons from intense laser-plasma interactions, Nature (London) 431, 535 (2004).

[6] C. Schroeder, E. Esarey, C. Geddes, C. Benedetti, and W. Leemans, Physics considerations for laser-plasma linear colliders, Phys. Rev. ST Accel. Beams 13, 101301 (2010).

[7] H. T. Kim, K. H. Pae, H. J. Cha, I. J. Kim, T. J. Yu, J. H. Sung, S. K. Lee, T. M. Jeong, and J. Lee, Enhancement of Electron Energy to the Multi-GeV Regime by a Dual-Stage Laser-Wakefield Accelerator Pumped by Petawatt Laser Pulses, Phys. Rev. Lett. 111, 165002 (2013).

[8] S. Steinke et al., Multistage coupling of independent laserplasma accelerators, Nature (London) 530, 190 (2016).

[9] J. Luo, M. Chen, W. Wu, S. Weng, Z. Sheng, C. Schroeder, D. Jaroszynski, E. Esarey, W. Leemans, W. Mori et al., Multistage Coupling of Laser-Wakefield Accelerators with Curved Plasma Channels, Phys. Rev. Lett. 120, 154801 (2018).

[10] K. Floettmann, Some basic features of the beam emittance, Phys. Rev. ST Accel. Beams 6, 034202 (2003).

[11] V. Malka, J. Faure, Y. A. Gauduel, E. Lefebvre, A. Rousse, and K. T. Phuoc, Principles and applications of compact laser-plasma accelerators, Nat. Phys. 4, 447 (2008).

[12] H.-P. Schlenvoigt et al., A compact synchrotron radiation source driven by a laser-plasma wakefield accelerator, Nat. Phys. 4, 130 (2008).

[13] S. Kneip et al., X-ray phase contrast imaging of biological specimens with femtosecond pulses of betatron radiation from a compact laser plasma wakefield accelerator, Appl. Phys. Lett. 99, 093701 (2011).

[14] M. Reiser, Theory and Design of Charged Particle Beams (Wiley, New York, 2008).

[15] T. O. Raubenheimer, Estimates of emittance dilution and stability in high-energy linear accelerators, Phys. Rev. ST Accel. Beams 3, 121002 (2000).

[16] M. Migliorati, A. Bacci, C. Benedetti, E. Chiadroni, M. Ferrario, A. Mostacci, L. Palumbo, A. Rossi, L. Serafini, and P. Antici, Intrinsic normalized emittance growth in laser-driven electron accelerators, Phys. Rev. ST Accel. Beams 16, 011302 (2013).

[17] T. Mehrling, J. Grebenyuk, F. Tsung, K. Floettmann, and J. Osterhoff, Transverse emittance growth in staged laser-wakefield acceleration, Phys. Rev. ST Accel. Beams 15, 111303 (2012).

[18] T. Kanai, A. Suda, S. Bohman, M. Kaku, S. Yamaguchi, and K. Midorikawa, Pointing stabilization of a highrepetition-rate high-power femtosecond laser for intense few-cycle pulse generation, Appl. Phys. Lett. 92, 061106 (2008).

[19] K. Nakamura, H.-S. Mao, A. J. Gonsalves, H. Vincenti, D. E. Mittelberger, J. Daniels, A. Magana, C. Toth, and W. P. Leemans, Diagnostics, control and performance parameters for the BELLA high repetition rate petawatt class laser, IEEE J. Quantum Electron. 53, 1 (2017).

[20] M. Thévenet, D. E. Mittelberger, K. Nakamura, A. J. Gonsalves, C. Benedetti, S. Steinke, J. Daniels, R. Lehe, C. B. Schroeder, J.-L. Vay, E. Esarey, and W. P. Leemans (to be published).

[21] A. Gonsalves, K. Nakamura, J. Daniels, H.-S. Mao, C. Benedetti, C. Schroeder, C. Tóth, J. van Tilborg, D. Mittelberger, S. Bulanov et al., Generation and pointing stabilization of multi-GeV electron beams from a laser plasma accelerator driven in a pre-formed plasma waveguide, Phys. Plasmas 22, 056703 (2015).

[22] P. Sprangle, E. Esarey, J. Krall, and G. Joyce, Propagation and Guiding of Intense Laser Pulses in Plasmas, Phys. Rev. Lett. 69, 2200 (1992).

[23] K. Nakamura, H. S. Mao, A. J. Gonsalves, H. Vincenti, D. E. Mittelberger, J. Daniels, A. Magana, C. Toth, and W. P. Leemans, Diagnostics, control and performance parameters for the BELLA high repetition rate petawatt class laser, IEEE J. Quantum Electron. 53, 1 (2017).

[24] J.-L. Vay, C. Geddes, E. Esarey, C. Schroeder, W. Leemans, E. Cormier-Michel, and D. Grote, Modeling of $10 \mathrm{GeV}-1$ $\mathrm{TeV}$ laser-plasma accelerators using Lorentz boosted simulations, Phys. Plasmas 18, 123103 (2011).

[25] J.-L. Vay, D. Grote, R. Cohen, and A. Friedman, Novel methods in the Particle-In-Cell accelerator Code-Framework Warp, Comput. Sci. Discovery 5, 014019 (2012).

[26] J.-L. Vay, Noninvariance of Space- and Time-Scale Ranges under a Lorentz Transformation and the Implications for the Study of Relativistic Interactions, Phys. Rev. Lett. 98, 130405 (2007).

[27] J. B. Cole, A high-accuracy realization of the Yee algorithm using non-standard finite differences, IEEE Trans. Microwave Theory Tech. 45, 991 (1997); High-accuracy Yee algorithm based on nonstandard finite differences: new developments and verifications, IEEE Trans. Antennas Propag. 50, 1185 (2002); M. Karkkainen, E. Gjonaj, T. Lau, and T. Weiland, in Computational accelerator physics. Proceedings, 9th International Conference, ICAP 06, Charmonix, France, 2006 (CERN, Geneva, Switzerland, 2006), pp. 35-40.

[28] B. M. Cowan, D. L. Bruhwiler, E. Cormier-Michel, E. Esarey, C. G. Geddes, P. Messmer, and K. M. Paul, Characteristics of an envelope model for laser-plasma accelerator simulation, J. Comput. Phys. 230, 61 (2011).

[29] B. B. Godfrey and J.-L. Vay, Suppressing the numerical Cherenkov instability in FDTD PIC codes, J. Comput. Phys. 267, 1 (2014)..

[30] P. Michel, C. Schroeder, B. Shadwick, E. Esarey, and W. Leemans, Radiative damping and electron beam dynamics 
in plasma-based accelerators, Phys. Rev. E 74, 026501 (2006).

[31] L. M. Gorbunov and V. I. Kirsanov, Excitation of plasma waves by an electromagnetic wave packet, Sov. Phys. JETP 66, 290 (1987).

[32] N. Andreev, E. Chizhonkov, A. Frolov, and L. Gorbunov, On laser wakefield acceleration in plasma channels, Nucl. Instrum. Methods Phys. Res., Sect. A 410, 469 (1998).

[33] R. J. England, R. J. Noble, K. Bane, D. H. Dowell, C.-K. Ng, J. E. Spencer, S. Tantawi, Z. Wu, R. L. Byer, E. Peralta et al., Dielectric laser accelerators, Rev. Mod. Phys. 86, 1337 (2014).

[34] P. Chen, J. Dawson, R. W. Huff, and T. Katsouleas, Acceleration of Electrons by the Interaction of a Bunched Electron Beam with a Plasma, Phys. Rev. Lett. 54, 693 (1985).

[35] R. Lehe, C. Thaury, E. Guillaume, A. Lifschitz, and V. Malka, Laser-plasma lens for laser-wakefield accelerators, Phys. Rev. ST Accel. Beams 17, 121301 (2014).
[36] C. Thaury, E. Guillaume, A. Döpp, R. Lehe, A. Lifschitz, K. T. Phuoc, J. Gautier, J.-P. Goddet, A. Tafzi, A. Flacco et al., Demonstration of relativistic electron beam focusing by a laser-plasma lens, Nat. Commun. 6, 6860 (2015).

[37] J. Rosenzweig, A. Cook, A. Scott, M. Thompson, and R. Yoder, Effects of Ion Motion in Intense Beam-Driven Plasma Wakefield Accelerators, Phys. Rev. Lett. 95, 195002 (2005).

[38] C. Benedetti, C. Schroeder, E. Esarey, and W. Leemans, Emittance preservation in plasma-based accelerators with ion motion, Phys. Rev. Accel. Beams 20, 111301 (2017).

[39] G. Dugan, Advanced accelerator system requirements for future linear colliders, AIP Conf. Proc. 737, 29 (2004).

[40] N. Merminga, P. Morton, J. Seeman, and W. Spence, Transverse phase space in the presence of dispersion, Stanford Linear Accelerator Center Technical Report No. SLAC-PUB-5514, 1991.

[41] A. W. Chao, Physics of Collective Beam Instabilities in High Energy Accelerators (Wiley, New York, 1993). 